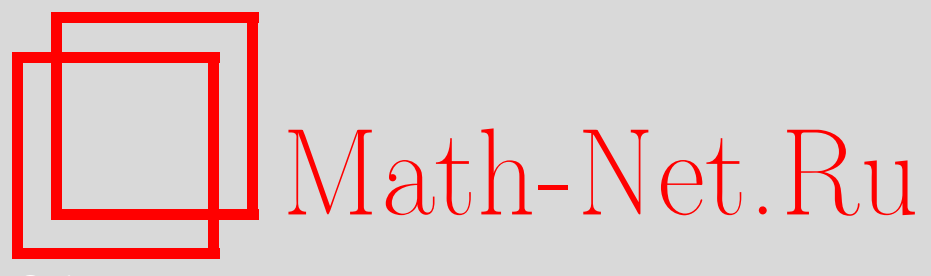

Ю. Н. Дрожжинов, Б. И. Завьялов, Асимптотически однородные обобщенные функции по специальным группам преобразований, Матем. сб., 2009, том 200, номер 6, 23-66

DOI: https://doi.org/10.4213/sm5656

Использование Общероссийского математического портала Math-Net.Ru подразумевает, что вы прочитали и согласны с пользовательским соглашением http://www .mathnet.ru/rus/agreement

Параметры загрузки:

IP: 54.84 .234 .179

26 апреля 2023 г., 16:16:17

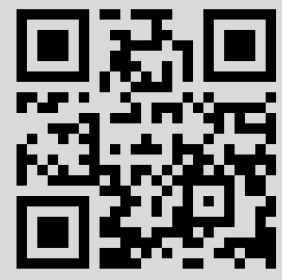




\author{
Ю. Н. Дрожжинов, Б. И. Завьялов
}

\title{
Асимптотически однородные обобщенные функции по специальным группам преобразований
}

Обобщенные функции, обладающие (квази)асимптотикой по специальным группам преобразований аргументов этих функций в асимптотической шкале правильно меняющихся функций, называются асимптотически однородными по этим группам преобразований. В частности, к этим функциям принадлежат все "квазиоднородные" обобщенные функции. В работе получено полное описание асимптотически однородных функций по группе преобразований, определяемой вектором $a \in \mathbb{R}_{+}^{n}$, в том числе и для случая критических порядков. Для этого вводятся и изучаются специальные пространства обобщенных функций. Полученные результаты применяются для изучения особенностей голоморфных функций в трубчатых областях над ортантами.

Библиография: 10 названий.

Ключевые слова: обобщенные функции, тауберовы теоремы, голоморфные функции.

\section{Введение}

Пусть $\mathscr{N}$ - некоторое пространство (основных) функций (например, $\mathscr{S}\left(\mathbb{R}^{n}\right)$, $\mathscr{D}\left(\mathbb{R}^{n}\right)$ и т.п. $), \mathscr{N}^{\prime}$ - пространство обобщенных функций, т.е. пространство линейных непрерывных функционалов над пространством соответствующих основных функций. Следуя стандартам, принятым в теории обобщенных функций, будем указывать обобщенную функцию $f \in \mathscr{N}^{\prime}$ вместе с аргументом основной функции и будем писать $(f(t), \psi(t)), \psi(t) \in \mathscr{N}$, вместо $(f, \psi)$. В такой записи $f(U t)$, где $U$ - линейное преобразование координат, означает обобщенную функцию, действующую по правилу

$$
(f(U t), \psi(t))=\frac{1}{\operatorname{det} U}\left(f(t), \psi\left(U^{-1} t\right)\right) .
$$

ОПРЕДЕЛЕНИЕ 0.1. Пусть $\left\{U_{k}, k>0\right\}$ - мультипликативная, непрерывная, однопараметрическая группа линейных преобразований $\mathbb{R}^{n}$ такая, что $U_{k_{1} k_{2}}=$ $U_{k_{1}} U_{k_{2}}$. Пусть $\mathscr{M}$ - подпространство $\mathscr{N}$, причем $\mathscr{M}$ и $\mathscr{N}$ инвариантны относительно $U_{k}, \rho(k)>0$ - непрерывная функция при $k>0$ и $f \in \mathscr{N}^{\prime}$. Будем говорить, что $f$ обладает квазиасимптотикой относителъно $\rho(k)$ nо группе $U_{k}$

Работа выполнена при поддержке Российского фонда фундаментальных исследований (грант № 07-01-00144a) и Программы поддержки ведущих научных школ РФ (грант № НШ-3224.2008.1).

(C) Ю. Н. Дрожжинов, Б. И. ЗАвьялов, 2009 
на $\mathscr{M}$, если для любой $\psi(t) \in \mathscr{M}$ и некоторой $g \in \mathscr{N}^{\prime}$

$$
\frac{1}{\rho(k)}\left(f\left(U_{k} t\right), \psi(t)\right) \underset{k \rightarrow \infty}{\longrightarrow}(g(t), \psi(t)) .
$$

Если $\mathscr{M}$ совпадает со всем пространством $\mathscr{N}$, то будем говорить, что $f$ асимптотически однородна по группе преобразований $\left\{U_{k}, k>0\right\}$.

Отметим, что $U_{k}$ может быть представлена в виде $U_{k}=e^{E \ln k}$, где $E$ - некоторое линейное преобразование $\mathbb{R}^{n}$. Далее мы ограничимся следующим важным для приложений случаем, когда вещественная жорданова форма $E$ имеет диагональный вид, причем все диагональные элементы либо положительны, либо отрицательны. Таким образом, если $a=\left(a_{1}, \ldots, a_{n}\right) \in \mathbb{R}_{+}^{n}$, то можно выбрать координаты $t=\left(t_{1}, \ldots, t_{n}\right)$ так, что

$$
U_{k}^{a} t=\left(k^{a_{1}} t_{1}, \ldots, k^{a_{n}} t_{n}\right), \quad U_{1 / k}^{a} t=U_{k}^{-a} t=\left(\frac{t_{1}}{k^{a_{1}}}, \ldots, \frac{t_{n}}{k^{a_{n}}}\right) .
$$

ОПРЕДЕЛЕНИЕ 0.2. В соответствии с определением 0.1 будем говорить, что $f(t) \in \mathscr{N}^{\prime}$ обладает квазиасимптотикой по группе преобразований, определяемой вектором $a \in \mathbb{R}_{+}^{n}$, на бесконечности (в нуле) относительно $\rho(k)$ на множестве $\mathscr{M}$, если для любой $\psi \in \mathscr{M}$

$$
\begin{gathered}
\frac{1}{\rho(k)}\left(f\left(U_{k}^{a} t\right), \psi(t)\right) \underset{k \rightarrow \infty}{\longrightarrow}(g(t), \psi(t)) \\
\left(\frac{1}{\rho(k)}\left(f\left(U_{k}^{-a} t\right), \psi(t)\right) \underset{k \rightarrow \infty}{\longrightarrow}(g(t), \psi(t))\right)
\end{gathered}
$$

для некоторой $g \in \mathscr{N}^{\prime}$. Если $\mathscr{M}=\mathscr{N}$, то будем говорить, что $f$ aсuмnтотически однородна по группе преобразований, определяемой вектором $а \in \mathbb{R}_{+}^{n}$, на бесконечности (в нуле) относителъно $\rho(k)$. Класс таких обобщенных функций обозначим через $A O_{\rho}^{a}(\mathscr{N})\left(A O_{\rho}^{-a}(\mathscr{N})\right.$ соответственно).

Если $g=0$, то будем говорить, что $f(t)$ обладает тривиальной квазиасимптотикой по группе преобразований, определяемой вектором $а \in \mathbb{R}_{+}^{n}$, на бесконечности (в нуле) относительно $\rho(k)$.

Если для $f \in \mathscr{N}^{\prime}$ выполнено соотношение $(0.1)$ и $g \neq 0$, то функция $\rho(k)$ обязательно является автомодельной (правильно меняющейся) функцией (см. [1]).

Напомним, что положительная непрерывная функция $\rho(k)$ при $k>0$ называется автомодельной, если для любого $a>0$ и некоторого $\alpha \in \mathbb{R}$ при $k \rightarrow \infty$

$$
\frac{\rho(a k)}{\rho(k)} \rightarrow a^{\alpha}
$$

равномерно на компактах по $а$ на $(0,+\infty)$. Число $\alpha$ называется порядком автомодельности $\rho$. Порядок $\alpha$ автомодельной функции $\rho(k)$ из $(0.1)$ называется порядком асимптотически однородной обобщенной функции.

Заметим, что если $\rho(k)$ в соотношении $(0.1)$ имеет порядок $\alpha$ и $\mathscr{M}=\mathscr{N}$, то $g$ является однородной обобщенной функцией степени $\alpha$ по группе преобразований аргумента, определяемой вектором $a$, т.е. $g\left(U_{k}^{a} t\right)=k^{\alpha} g(t), k>0$. Такие функции называют иногда "квазиоднородными" (см. [2]). 
Отметим следующее свойство автомодельных функций.

Лемма 0.1. Пусть $\rho(k)$ - автомоделъная функиия порядка $\alpha$. Тогда для любого $\varepsilon>0$ существует константа с такая, что

$$
\begin{cases}\frac{1}{c} t^{\alpha-\varepsilon} \leqslant \frac{\rho(k t)}{\rho(k)} \leqslant c t^{\alpha+\varepsilon} & \text { nрu } k>1, t>1, \\ \frac{1}{c} t^{\alpha+\varepsilon} \leqslant \frac{\rho(k t)}{\rho(k)} \leqslant c t^{\alpha-\varepsilon} & \text { npu } \frac{1}{k}<t<1, k>1 .\end{cases}
$$

Пусть $\rho(k)$ - автомодельная функция порядка $\alpha$. Введем функцию

$$
\widehat{\rho}(k)= \begin{cases}k^{\alpha} \int_{1}^{k} \frac{\rho(\varkappa)}{\varkappa^{\alpha+1}} d \varkappa, & \text { если } \int_{1}^{\infty} \frac{\rho(\varkappa)}{\varkappa^{\alpha+1}} d \varkappa=\infty, \\ k^{\alpha} \int_{k}^{\infty} \frac{\rho(\varkappa)}{\varkappa^{\alpha+1}} d \varkappa, & \text { если } \int_{1}^{\infty} \frac{\rho(\varkappa)}{\varkappa^{\alpha+1}} d \varkappa<\infty .\end{cases}
$$

Нетрудно видеть, что $\widehat{\rho}(k)$ - тоже автомодельная функция порядка $\alpha$. Отметим некоторые ее свойства.

1) Пусть непрерывная комплекснозначная функция $r(k) \underset{k \rightarrow+\infty}{\longrightarrow} 1$. Тогда

$$
\begin{array}{ll}
\frac{k^{\alpha}}{\widehat{\rho}(k)} \int_{1}^{k} \frac{\rho(\varkappa) r(\varkappa)}{\varkappa^{\alpha+1}} d \varkappa \underset{k \rightarrow+\infty}{\longrightarrow} 1, & \text { если } \int_{1}^{\infty} \frac{\rho(\varkappa)}{\varkappa^{\alpha+1}} d \varkappa=\infty, \\
\frac{k^{\alpha}}{\widehat{\rho}(k)} \int_{k}^{\infty} \frac{\rho(\varkappa) r(\varkappa)}{\varkappa^{\alpha+1}} d \varkappa \underset{k \rightarrow+\infty}{\longrightarrow} 1, \quad \text { если } \int_{1}^{\infty} \frac{\rho(\varkappa)}{\varkappa^{\alpha+1}} d \varkappa<\infty .
\end{array}
$$

2) Имеет место следующая сходимость:

$$
\frac{\widehat{\rho}(k)}{k^{\alpha}} \underset{k \rightarrow+\infty}{\longrightarrow} \begin{cases}+\infty, & \text { если } \int_{1}^{\infty} \frac{\rho(\varkappa)}{\varkappa^{\alpha+1}} d \varkappa=\infty, \\ 0, & \text { если } \int_{1}^{\infty} \frac{\rho(\varkappa)}{\varkappa^{\alpha+1}} d \varkappa<\infty .\end{cases}
$$

3) Справедливо соотношение

$$
\frac{\widehat{\rho}(k)}{\rho(k)} \underset{k \rightarrow+\infty}{\longrightarrow}+\infty .
$$

Асимптотически однородные функции обладают рядом интересных свойств и используются в формулировках многих тауберовых теорем, а также в различных задачах математической физики. Их описание и свойства для случая $a \in \mathbb{R}_{+}^{1}$ хорошо изучены для функций из $\mathscr{S}_{+}^{\prime}-$ пространства обобщенных функций медленного роста с носителями на положительной полуоси (см. [3], [4]). Пространство $\mathscr{S}_{+}^{\prime}$ является сопряженным к пространству $\mathscr{S}_{+}$, состоящему из бесконечно дифференцируемых функций на $[0,+\infty)$, убывающих на бесконечности вместе со всеми производными быстрее любой отрицательной степени $t$. В частности, если $f(t) \in \mathscr{S}_{+}^{\prime}$ асимптотически однородна относительно автомодельной функции $\rho(k)$ порядка $\alpha$, то в $\mathscr{S}_{+}^{\prime}$

$$
\frac{1}{\rho(k)} f(k t) \underset{k \rightarrow+\infty}{\longrightarrow} C f_{\alpha+1}(t),
$$


где ядро дробного (дифференцирования) интегрирования $f_{\beta}(t) \in \mathscr{S}_{+}^{\prime}$ определяется формулой

$$
f_{\beta}(t)= \begin{cases}\frac{\Theta(t) t^{\beta-1}}{\Gamma(\beta)} & \text { при } \beta>0, \\ \frac{d^{N}}{d t^{N}} f_{\beta+N}(t) & \text { при } \beta \leqslant 0, \beta+N>0, \text { рекуррентно по } N .\end{cases}
$$

Здесь $\Gamma(\beta)$ - гамма-функция, $\Theta(t)$ - функция Хевисайда (характеристическая функция положительной полуоси $\left.\mathbb{R}_{+}\right)$.

Для того чтобы $f(t) \in \mathscr{S}_{+}^{\prime}$ была асимптотически однородна относительно автомодельной функции $\rho(k)$ порядка $\alpha$, необходимо и достаточно, чтобы существовало такое число $N>-\alpha-1$, что

$$
\frac{f^{(-N)}(t)}{t^{N} \rho(t)} \underset{t \rightarrow+\infty}{\longrightarrow} A .
$$

Здесь $f^{(-N)}(t)$ - первообразная порядка $N$.

Первообразная (производная) порядка $\beta$ обобщенной функиии $f(t) \in \mathscr{S}_{+}^{\prime}\left(\mathbb{R}^{1}\right)$ определяется формулой

$$
f^{(-\beta)}(t)=f_{\beta}(t) * f(t)
$$

Как известно,

$$
\frac{N !}{t^{N}} f^{(-N)}(t)=S_{r}^{N}(f)
$$

- чезаровское среднее порядка $N$. Таким образом, понятие асимптотической однородности в случае $\mathscr{S}_{+}^{\prime}$ адекватно отражает асимптотическое поведение чезаровских средних (см. [4]).

Основная цель настоящей статьи - получить полное описание асимптотически однородных функций на бесконечности по группе преобразований, определяемой вектором $a \in \mathbb{R}_{+}^{n}$, в общем случае. Для получения этого описания используется так называемое обобщенное сферическое представление обобщенных функций, введенное в 1 . Это представление сводит изучение асимптотических свойств обобщенных функций по группе $U_{k}^{a}$ к исследованию радиальных асимптотических свойств обобщенных функций, заданных на специальных пространствах основных функций. Асимптотически однородные обобщенные функции на этих специальных пространствах изучаются в $\S 2, \S 3$. Основные теоремы работы будут изложены в $\S 4$.

Наконец, в 55 мы рассмотрим применение полученных результатов в исследованиях связи асимптотически однородных обобщенных функции из $\mathscr{S}^{\prime}\left(\mathbb{R}^{n}\right)$ с особенностями функций, голоморфных в трубчатых областях, сумма граничных значений которых представляет эти обобщенные функции.

\section{§ 1. Обобщенное сферическое представление обобщенных функций}

Пусть $S^{n-1}$ - единичная сфера в $\mathbb{R}^{n}$ и задан вектор $a=\left(a_{1}, \ldots, a_{n}\right) \in \mathbb{R}_{+}^{n}$ (т.е. $\left.a_{i}>0, i=1, \ldots, n\right)$. Сопоставим каждой функции $\varphi(t) \in \mathscr{S}\left(\mathbb{R}^{n}\right)$ функцию

$$
\tau_{a}[\varphi(t)]=\varphi\left(r^{a_{1}} e_{1}, \ldots, r^{a_{n}} e_{n}\right)=\psi(r, e),
$$


где $r \in \mathbb{R}_{+}$и $e=\left(e_{1}, \ldots, e_{n}\right) \in S^{n-1}$. Это отображение, в сущности, есть обобщенная сферическая замена переменных со степенной особенностью в начале координат:

$$
\mu(r, e)=t: \quad t_{i}=r^{a_{i}} e_{i}, \quad i=1, \ldots, n, \quad e=\left(e_{1}, \ldots, e_{n}\right) \in S^{n-1}, \quad r>0 .
$$

Для того чтобы описать образ отображения $\tau_{a}$ и обосновать соответствующую замену переменных для обобщенных функций, мы введем специальные пространства основных и обобщенных функций.

Пусть $J$ - не более чем счетное (может быть, пустое) множество вещественных чисел $\lambda$ без точек накопления, ограниченное снизу. Такие множества вещественных чисел будем называть допустимыми.

Обозначим через $\mathscr{S}_{J}$ пространство функций $\psi(r) \in C^{\infty}\left(\mathbb{R}_{+}\right)$, быстро убывающих при $r \rightarrow+\infty$ вместе со всеми производными и таких, что для некоторых постоянных $C_{\lambda}$, зависящих от $\psi$,

$$
\left[\psi(r)-\sum_{\substack{\lambda \leqslant N \\ \lambda \in J}} C_{\lambda} r^{\lambda}\right] \in C^{N}([0,+\infty)),\left.\quad\left(\frac{d}{d r}\right)^{\ell}\left[\psi(r)-\sum_{\substack{\lambda \leqslant N \\ \lambda \in J}} C_{\lambda} r^{\lambda}\right]\right|_{r=0}=0,
$$

где $N \geqslant 0, \ell=0, \ldots, N$. Топологию на $\mathscr{S}_{J}$ зададим с помощью следующей системы норм:

$$
\mathscr{P}_{N}(\psi)=\max _{0 \leqslant \ell \leqslant N} \sup _{r>0}(1+r)^{N}\left|\left(\frac{d}{d r}\right)^{\ell}\left[\psi(r)-\sum_{\substack{\lambda \leqslant N \\ \lambda \in J}} C_{\lambda} r^{\lambda} \eta(r)\right]\right|+\max _{\substack{\lambda \leqslant N \\ \lambda \in J}}\left|C_{\lambda}\right| .
$$

Здесь и всюду далее функция $\eta(r)$ бесконечно дифференцируема на $[0,+\infty)$, финитна и $\eta(r)=1$ в некоторой окрестности нуля. Например,

$$
\eta(r) \in C^{\infty}([0,+\infty)), \quad \eta(r)= \begin{cases}1 & \text { при } r<1, \\ 0 & \text { при } r>2 .\end{cases}
$$

Ясно, что топология на $\mathscr{S}_{J}$ не зависит от выбора функции $\eta(r)$ с такими свойствами. Пространство $\mathscr{S}_{J}$ - пространство Фреше. Отметим также, что $\mathscr{S}_{J}$ инвариантно относительно растяжений аргумента функций из этого пространства. Кроме того, если множество $J^{\prime}$ содержит $J$, то $\mathscr{S}_{J} \subset \mathscr{S}_{J^{\prime}}$. (Для добавочных точек $\lambda \in J^{\prime}$, но не принадлежащих $J$, считаем, что $C_{\lambda}=0$.) В частности, если $J=\varnothing$ (обозначим его через $\left.J_{\varnothing}\right)$, то $\mathscr{S}_{J_{\varnothing}} \subset \mathscr{S}_{J}$ для любого допустимого множества $J$ и состоит из функций из $\mathscr{S}_{+}$, имеющих нуль бесконечного порядка в нуле.

Пространство $\mathscr{S}\left(S^{n-1}\right)=C^{\infty}\left(S^{n-1}\right)$ - пространство бесконечно дифференцируемых на $S^{n-1}$ функций со стандартной топологией равномерной сходимости вместе со всеми производными. Точнее, пусть $e=\left(e_{1}, \ldots, e_{n}\right) \in S^{n-1}$ и $U_{i}, i=1,2, \ldots, M$, - атлас карт на единичной сфере, в каждой карте $\Theta=$ $\left(\theta_{1}, \ldots, \theta_{n-1}\right)-$ соответствующие координаты. Положим

$$
Q_{N}^{i}\{\psi(e)\}=\max _{|j| \leqslant N} \sup _{\Theta \in U_{i}}\left|\frac{\partial^{|j|}}{\partial \theta_{1}^{j_{1}} \cdots \partial \theta_{n-1}^{j_{n-1}}} \psi(e(\Theta))\right|, \quad e \in U_{i} .
$$


Как обычно, если $j=\left(j_{1}, \ldots, j_{n}\right)$ - мультииндекс, то

$$
j !=j_{1} ! \cdots j_{n} !, \quad|j|=j_{1}+\cdots+j_{n}, \quad \varphi^{(j)}(x) \equiv D^{j} \varphi(x)=\frac{\partial^{|j|}}{\partial x_{1}^{j_{1}} \cdots \partial x_{n}^{j_{n}}} \varphi(x) .
$$

В качестве системы полунорм в $\mathscr{S}\left(S^{n-1}\right)$ возьмем

$$
Q_{N}\{\psi(e)\}=\max _{i} Q_{N}^{i}\{\psi(e)\}, \quad N=0,1, \ldots
$$

Очевидно, что эта топология не зависит от выбора атласа на единичной сфере.

Обозначим через $\mathscr{S}^{N}\left(S^{n-1}\right)$ пополнение $C^{\infty}\left(S^{n-1}\right)$ по норме $Q_{N}$. Тогда $\mathscr{S}\left(S^{n-1}\right) \subset \mathscr{S}^{N}\left(S^{n-1}\right)$.

Положим $W_{J}=\mathscr{S}_{J} \otimes \mathscr{S}\left(S^{n-1}\right)$ - проективное тензорное произведение пространств $\mathscr{S}_{J}$ и $\mathscr{S}\left(S^{n-1}\right)$ (см. [5]). Пространство $W_{J}$ может быть реализовано как пространство функций $\psi(r, e), r \in \mathbb{R}_{+}, e \in S^{n-1}$, бесконечно дифференцируемых при $e \in S^{n-1}$ и $r \in \mathbb{R}_{+}=\{r>0\}$, таких, что

$$
\begin{gathered}
\psi(r, e)-\sum_{\substack{\lambda \in J \\
\lambda \leqslant N}} C_{\lambda}(e) r^{\lambda} \in C^{N}\left([0,+\infty) \times \mathscr{S}\left(S^{n-1}\right)\right), \\
\left.\left(\frac{d}{d r}\right)^{\ell}\left[\psi(r, e)-\sum_{\substack{\lambda \leqslant N \\
\lambda \in J}} C_{\lambda}(e) r^{\lambda}\right]\right|_{r \rightarrow+0}=0, \quad 0 \leqslant \ell \leqslant N, \quad N=0,1, \ldots .
\end{gathered}
$$

Здесь $C_{\lambda}(e) \in \mathscr{S}\left(S^{n-1}\right), \lambda \in J$. Топология на $W_{J}$ задается с помощью следующей системы (полу)норм

$$
\begin{gathered}
\mathscr{P}_{N}(\psi)=\max _{0 \leqslant \ell \leqslant N} \sup _{r>0} Q_{N}\left\{(1+r)^{N}\left(\frac{d}{d r}\right)^{\ell}\left[\psi(r, e)-\sum_{\substack{\lambda \leqslant N \\
\lambda \in J}} C_{\lambda}(e) r^{\lambda} \eta(r)\right]\right\} \\
+\max _{\lambda \leqslant N, \lambda \in J} Q_{N}\left\{C_{\lambda}(e)\right\},
\end{gathered}
$$

где функция $\eta(r)$ удовлетворяет условию (1.5). Заметим, что топология на $W_{J}$ не зависит от выбора функции $\eta(r)$ с такими свойствами. Таким образом,

$$
W_{J} \subset \cdots \subset W_{J}^{N+1} \subset W_{J}^{N} \subset \cdots \subset W_{J}^{0},
$$

где $W_{J}^{N}$ - банахово пространство с нормой $\mathscr{P}_{N}(\psi)$ (пополнение $W_{J}$ по норме $\left.\mathscr{P}_{N}\right)$.

Сопоставим теперь каждому $\lambda \in J$ линейное замкнутое подпространство $\mathscr{E}_{\lambda} \in \mathscr{S}\left(S^{n-1}\right)$ и положим

$$
\mathscr{F}=\left\{\mathscr{E}_{\lambda}, \lambda \in J\right\}
$$

Определим в пространстве $W_{J}$ подпространство

$$
V_{J, \mathscr{F}}=\left\{\psi(r, e) \in W_{J}: C_{\lambda}(e) \in \mathscr{E}_{\lambda}, \mathscr{E}_{\lambda} \in \mathscr{F}\right\} .
$$

Топология в $V_{J, \mathscr{F}}$ наследуется топологией в $W_{J}$. 
Пусть задан вектор $a=\left(a_{1}, \ldots, a_{n}\right) \in \mathbb{R}_{+}^{n}$. Обозначим через $J_{a}$ допустимое множество

$$
J_{a} \equiv J=\left\{\lambda=m_{1} a_{1}+\cdots+m_{n} a_{n} ; m_{i}=0,1,2, \ldots ; i=1,2, \ldots, n\right\} .
$$

Пространства, соответствующие этому множеству, обозначим через $\mathscr{S}_{J_{a}}$ и $W_{J_{a}}$. Положим

$$
E_{j}(e)=e_{1}^{j_{1}} \cdots e_{n}^{j_{n}} \in \mathscr{S}\left(S^{n-1}\right) .
$$

Обозначим через $\mathscr{E}_{\lambda}^{a}$ линейную оболочку функций $E_{j}(e)$, у которых мультииндекс $j$ удовлетворяет уравнению $(j, a)=\lambda$, т.е.

$$
\mathscr{E}_{\lambda}^{a}=\operatorname{Lin}\left\{E_{j}(e),(j, a)=\lambda \in J_{a}\right\}
$$

Отметим, что функции $E_{j}(e) \in \mathscr{E}_{\lambda}$ линейно независимы и поэтому образуют базис в $\mathscr{E}_{\lambda} a$. Положим теперь $\mathscr{F}_{a}=\left\{\mathscr{E}_{\lambda} a, \lambda \in J_{a}\right\}$.

Введем согласно (1.9) пространство

$$
V_{a}=V_{J_{a}, \mathscr{F}}
$$

Нетрудно видеть, что $V_{a}$ - замкнутое подпространство пространства $W_{J_{a}}$.

УтвеРЖДЕНИЕ 1.1. Отображение $\tau_{a}$, определяемое формулой, осуществляет изоморфизм пространств $\mathscr{S}\left(\mathbb{R}^{n}\right)$ и $V_{a}$.

ДокАзАтЕльство. Очевидно, что $\tau_{a}$ есть непрерывное отображение $\mathscr{S}\left(\mathbb{R}^{n}\right)$ в $W_{J_{a}}$. Ядро этого отображения тривиально. Легко заметить, что $\tau_{a}$ отображает $\mathscr{S}\left(\mathbb{R}^{n}\right)$ в $V_{a}$. Действительно, для тейлоровского разложения $\varphi(t) \in \mathscr{S}\left(\mathbb{R}^{n}\right)$ имеем

$$
\begin{aligned}
\varphi(t) \sim \sum_{j} \frac{\varphi^{(j)}(0)}{j !} t^{j} & =\sum_{j} \frac{\varphi^{(j)}(0)}{j !} E_{j}(e) r^{(a, j)}=\sum_{\lambda \in J_{a}} r^{\lambda} \sum_{(a, j)=\lambda} \frac{\varphi^{(j)}(0)}{j !} E_{j}(e) \\
& =\sum_{\lambda \in J_{a}} C_{\lambda}(e) r^{\lambda}, \quad r \sim 0,
\end{aligned}
$$

где

$$
C_{\lambda}(e)=\sum_{(a, j)=\lambda} \frac{\varphi^{(j)}(0)}{j !} E_{j}(e) \in \mathscr{E}_{\lambda}^{a} .
$$

Теперь достаточно доказать, что $\tau_{a}$ есть отображение $\mathscr{S}\left(\mathbb{R}^{n}\right)$ на $V_{a}$. Из этого, в частности, будет следовать, что отображение $\tau_{a}^{-1}-$ непрерывное отображение $V_{a}$ на $\mathscr{S}\left(\mathbb{R}^{n}\right)$.

Итак, пусть $\psi(r, e) \in V_{a}$. Достаточно показать, что $\psi\left(\mu^{-1}(t)\right) \in \mathscr{S}\left(\mathbb{R}^{n}\right)$, где $\mu$ определяется формулой (1.2). Ясно, что функция $\psi\left(\mu^{-1}(t)\right)$ бесконечно дифференцируема вне начала координат и убывает вместе со всеми производными быстрее любой степени $1 /|t|$ при $|t| \rightarrow+\infty$. Покажем, что у этой функции существуют все производные в окрестности начала координат, т.е. 
$\psi\left(\mu^{-1}(t)\right) \in C^{\infty}(\omega)$, где $\omega-$ некоторая окрестность начала координат. Положим

$$
\Phi_{M}(r, e)=\psi(r, e)-\triangle_{M}(r, e), \quad \Delta_{M}(r, e)=\sum_{\substack{\lambda \leqslant M \\ \lambda \in J_{a}}} C_{\lambda}(e) r^{\lambda},
$$

где $C_{\lambda}(e)$ определяется формулой (1.7). Ясно, что $\Phi_{M}(r, e) M$ раз дифференцируема при $r \geqslant 0, e \in S^{n-1}$, причем

$$
\left(\frac{d}{d r}\right)^{\ell} \Phi_{M}(0, e)=0, \quad \ell=0,1, \ldots, M .
$$

Поэтому $\Phi_{M}(r, e)$ имеет нуль в нуле по $r$ порядка $M$. Поэтому для любого $N$ существует $M$ такое, что $\Phi\left(\mu^{-1}(t)\right) \in C^{N}(\omega)$. Покажем, что $\triangle_{M}\left(\mu^{-1}(t)\right) \in C^{\infty}(\omega)$ для любого $M$. Действительно, рассмотрим произвольное слагаемое этой суммы, имеем

$$
\begin{aligned}
C_{\lambda}(e) r^{\lambda} & =\sum_{(j, a)=\lambda} b_{j} E_{j}(e) r^{\lambda}=\sum_{(j, a)=\lambda} b_{j} e_{1}^{j_{1}} \cdots e_{n}^{j_{n}} r^{a_{1} j_{1}+\cdots+j_{n} a_{n}} \\
& =\sum_{(j, a)=\lambda} b_{j}\left(r^{a_{1}} e_{1}\right)^{j_{1}} \cdots\left(r^{a_{n}} e_{n}\right)^{j_{n}}=\sum_{(j, a)=\lambda} b_{j} t^{j} .
\end{aligned}
$$

Отсюда следует, что $\psi\left(\mu^{-1}(t)\right) \in C^{N}(\omega)$. Так как $N$ произвольно, то $\psi\left(\mu^{-1}(t)\right) \in$ $C^{\infty}(\omega)$. Утверждение доказано.

ОПРЕДЕЛЕНиЕ 1.1. Пусть $f(t) \in \mathscr{S}^{\prime}\left(\mathbb{R}^{n}\right)$. Тогда обобщенная функция $f_{s}(r, e)$, определяемая формулой

$$
\left(f_{s}(r, e), \psi(r, e)\right)=(f(t), \varphi(t)), \quad \varphi(t)=\varphi(\mu(r, e))=\psi(r, e) \in V_{a},
$$

принадлежит $V_{a}^{\prime}$. По теореме Хана-Банаха мы можем продолжить $f_{s}$ на все пространство $W_{J_{a}}$. Обозначим это продолжение через $F(r, e)$ и назовем его обобщенным сферическим представлением обобщенной функции $f(t) \in \mathscr{S}^{\prime}$. Таким образом,

$$
(F(r, e), \psi(r, e))=\left(f_{s}, \psi(r, e)\right) \quad \forall \psi(r, e) \in V_{a} .
$$

Обобщенное сферическое представление $F(r, e)$ обобщенной функции $f \in \mathscr{S}^{\prime}$ определяется неоднозначно. Для того, чтобы описать множество всех таких представлений, введем в $\mathscr{S}_{J}$ и $W_{J}$ специальные обобщенные функции, обобщающие обычные дельта-функции и их производные

ОПРЕДЕЛЕНИЕ 1.2. Для каждого $\lambda \in J$ и любой $\Phi(e) \in \mathscr{S}^{\prime}\left(S^{n-1}\right)$ определим обобщенные функции $\triangle_{\lambda}(r) \in \mathscr{S}_{J}^{\prime}$ и $\triangle_{\lambda}(r) \otimes \Phi(e) \in W_{J}^{\prime}$ по формулам:

$$
\begin{gathered}
\left(\triangle_{\lambda}(r), \psi(r)\right)=C_{\lambda}, \quad \psi \in \mathscr{S}_{J}, \\
\left(\triangle_{\lambda}(r) \otimes \Phi(e), \psi(r, e)\right)=\left(\Phi(e), C_{\lambda}(e)\right), \quad \psi \in W_{J},
\end{gathered}
$$

где $C_{\lambda}$ берем из (1.4), а $C_{\lambda}(e) \in \mathscr{S}\left(S^{n-1}\right)$ - из (1.7).

Отметим, что $\Delta_{\lambda}(r)$ (как и $\left.\triangle_{\lambda}(r) \otimes \Phi(e)\right)$ - однородные обобщенные функции по $r$ степени $-(\lambda+1)$. 
Пусть теперь $F_{1}$ и $F_{2}$ - обобщенные сферические представления $f(t) \in \mathscr{S}^{\prime}$. Обозначая

$$
\Psi(r, e)=F_{1}(r, e)-F_{2}(r, e)
$$

для любой $\psi(r, e) \in V_{J_{a}, \mathscr{F}}$ имеем

$$
(\Psi(r, e), \psi(r, e))=0 .
$$

Ясно, что $\operatorname{supp} \Psi(r, e) \subset\{0\} \times S^{n-1}$. Поэтому существует такое $N$, что

$$
\Psi(r, e)=\sum_{\substack{\lambda \leqslant N \\ \lambda \in J_{a}}} \triangle_{\lambda}(r) \otimes \Phi_{\lambda}(e), \quad \text { где } \Phi_{\lambda}(e) \in \mathscr{S}^{\prime}\left(S^{n-1}\right) .
$$

Отсюда для любой $\psi(r, e) \in V_{a}$ имеем

$$
\begin{gathered}
(\Psi(r, e), \psi(r, e))=\left(\sum_{\substack{\lambda \leqslant N \\
\lambda \in J_{a}}} \Delta_{\lambda}(r) \otimes \Phi_{\lambda}(e), \psi(r, e)\right)=\sum_{\substack{\lambda \leqslant N \\
\lambda \in J_{a}}} \Phi_{\lambda}(e), C_{\lambda}(e) \\
=\sum_{\substack{\lambda \leqslant N \\
\lambda \in J_{a}}} b_{j}\left(\Phi_{\lambda}(e), E_{j}(e)\right)=0
\end{gathered}
$$

где $(a, j)=\lambda$. Отметим, что $b_{j}$ могут быть произвольными: для этого достаточно в окрестности начала координат положить

$$
\psi(r, e)=\sum_{\substack{\lambda \leqslant N \\ \lambda \in J_{a}}} r^{\lambda} \sum_{(a, j)=\lambda} b_{j} E_{j}(e)
$$

Учитывая произвольность $b_{j}$, из $(1.16)$ выводим, что $\left(\Phi_{\lambda}(e), E_{j}(e)\right)=0$ для $(a, j)=\lambda$.

Обобщенное сферическое представление для любой $f(t) \in \mathscr{S}^{\prime}\left(\mathbb{R}^{n}\right)$ в общем случае имеет следующий вид:

$$
F(r, e)+\sum_{\substack{\lambda \leqslant N \\ \lambda \in J_{a}}} \Delta_{\lambda}(r) \otimes \Phi_{\lambda}(e)
$$

где $N$ - некоторое натуральное число, а $F(r, e)$ - какое-то конкретное обобщенное сферическое представление, а обобщенные функции $\Phi_{\lambda}(e)$ удовлетворяют условиям ортогональности, т.е.

$$
\left(\Phi_{\lambda}(e), E_{j}(e)\right)=0, \quad(a, j)=\lambda .
$$

ОПРЕДЕЛЕНИЕ 1.3. Пусть $\rho(k)$ - автомодельная функция порядка $\beta$. Будем говорить, что $F(r) \in \mathscr{S}_{J}^{\prime}$ асимптотически однородна на бесконечности (в нуле) относительно $\rho(k)$, и писать $F(r) \in A O_{\rho}\left(\mathscr{S}_{J}\right)($ соответственно $F(r) \in$ $\left.A O_{\rho}^{-1}\left(\mathscr{S}_{J}\right)\right)$, если для любой $\psi(r) \in \mathscr{S}_{J}$

$$
\begin{gathered}
\frac{1}{\rho(k)}(F(k r), \psi(r)) \underset{k \rightarrow \infty}{\longrightarrow}(G(r), \psi(r)) \\
\left(\frac{1}{\rho(k)}\left(F\left(\frac{r}{k}\right), \psi(r)\right) \underset{k \rightarrow \infty}{\longrightarrow}(G(r), \psi(r))\right) .
\end{gathered}
$$


Аналогично, $F(r, e) \in W_{J}^{\prime}$ асимптотически однородна по $r$ на бесконечности (в нуле) относительно $\rho(k)$, если для любой $\psi(r, e) \in W_{J}$

$$
\begin{gathered}
\frac{1}{\rho(k)}(F(k r, e), \psi(r, e)) \underset{k \rightarrow \infty}{\longrightarrow}(G(r, e), \psi(r, e)) \\
\left(\frac{1}{\rho(k)}\left(F\left(\frac{r}{k}, e\right), \psi(r, e)\right) \underset{k \rightarrow \infty}{\longrightarrow}(G(r, e), \psi(r, e))\right) .
\end{gathered}
$$

При этом пишем $F(r, e) \in A O_{\rho}\left(W_{J}\right)$ (соответственно $F(r, e) \in A O_{\rho}^{-1}\left(W_{J}\right)$ ).

Для пространства $V_{J, \mathscr{F}}$ определение асимптотической однородности относительно $\rho(k)$ определяется аналогично. В этом случае будем писать $F(r, e) \in$ $A O_{\rho}\left(V_{J, \mathscr{F}}\right)$ (соответственно $F(r, e) \in A O_{\rho}^{-1}\left(V_{J, \mathscr{F}}\right)$ ).

Пусть $F(r, e)$ - обобщенное сферическое представление для обобщенной функции $f(t) \in \mathscr{S}^{\prime}\left(\mathbb{R}^{n}\right)$. Тогда

$$
(f(t), \varphi(t))=\left(F(r, e), \varphi\left(r^{a_{1}} e_{1}, \ldots, r^{a_{n}} e_{n}\right)\right) \quad \forall \varphi \in \mathscr{S}\left(\mathbb{R}^{n}\right) .
$$

Поэтому

$$
\begin{gathered}
\left(f\left(k^{a_{1}} t_{1}, \ldots, k^{a_{n}} t_{n}\right), \varphi(t)\right)=\frac{1}{k^{|a|}}\left(f(t), \varphi\left(\frac{t_{1}}{k^{a_{1}}}, \ldots, \frac{t_{n}}{k^{a_{n}}}\right)\right) \\
\quad=\frac{1}{k^{|a|}}\left(F(r, e), \varphi\left(\frac{r^{a_{1}} e_{1}}{k^{a_{1}}}, \ldots, \frac{r^{a_{n}} e_{n}}{k^{a_{n}}}\right)\right) \\
\quad=\frac{1}{k^{|a|-1}}\left(F(k r, e), \varphi\left(r^{a_{1}} e_{1}, \ldots, r^{a_{n}} e_{n}\right)\right) .
\end{gathered}
$$

Отсюда следует

УТВЕРЖДЕНИЕ 1.2. Пусть $\rho(k)$ - автомоделъная функиия порядка $\alpha$ и вектор $а \in \mathbb{R}_{+}^{n}$. Для того чтобы обобщенная функиия $f(t) \in \mathscr{S}^{\prime}\left(\mathbb{R}^{n}\right)$ была асимптотически однородна на бесконечности относительно $\rho(k)$ по группе преобразований, определяемой вектором $a \in \mathbb{R}_{+}^{n}$, m.е. $f(t) \in A O_{\rho}^{a}\left(\mathscr{S}\left(\mathbb{R}^{n}\right)\right)$, необходимо и достаточно, чтобы ее обобщенное сферическое представление $F(r, e)$ бъло асимптотически однородным на бесконечности относительно $\rho_{1}(k)$ на $V_{a}$, m.е. $F(r, e) \in A O_{\rho_{1}}\left(V_{a}\right)$, где автомодельная функция $\rho_{1}(k)=k^{|a|-1} \rho(k)$ имеет порядок $\beta=\alpha+|a|-1$.

Таким образом, для описания класса $A O_{\rho}^{a}\left(\mathscr{S}\left(\mathbb{R}^{n}\right)\right)$ достаточно описать класс асимптотически однородных обобщенных функций на пространствах $V_{a} \subset W_{J_{a}}$, что в свою очередь требует описания асимптотически однородных обобщенных функций на пространстве $\mathscr{S}_{J_{a}}$.

\section{§ 2. Асимптотически однородные обобщенные функции на $\mathscr{S}_{J}$}

Пространство основных функций $\mathscr{S}_{J}$, где $J$ - допустимое множество, введено в $\S 1$ (см. (1.3)-(1.5)). Дадим несколько определений.

ОПРЕДЕЛЕНИЕ 2.1. Пусть $F(r) \in \mathscr{S}_{J}^{\prime}$ и имеет компактный носитель. Для $\lambda \in J$ определим моменты порядка $\lambda$ по формуле

$$
M_{\lambda}[F]=\left(F(r), r^{\lambda} \eta(r)\right)
$$


где $\eta(r) \in \mathscr{S}_{+}$и $\eta(r)=1$ в окрестности $\operatorname{supp} F$. Ясно, что $M_{\lambda}[F]$ не зависит от выбора $\eta(r)$ с такими свойствами.

Аналогично, для $F(r, e) \in W_{J}^{\prime}$ с компактным носителем момент no $r$ порядка $\lambda$ определяем формулой

$$
M_{\lambda}[F](e)=\left(F(r, e), r^{\lambda} \eta(r)\right)
$$

Обобщенную функцию $r_{J}^{\beta}$ определим формулой

$$
\left(r_{J}^{\beta}, \varphi(r)\right)=\left\{\begin{array}{l}
\int_{0}^{\infty} r^{\beta}\left(\varphi(r)-\sum_{\substack{\lambda \in J \\
\lambda<-\beta-1}} C_{\lambda} r^{\lambda}\right) d r, \quad \text { если }-\beta-1 \notin J, \\
\int_{0}^{\infty} r^{\beta}\left(\varphi(r)-\sum_{\substack{\lambda \in J \\
\lambda<-\beta-1}} C_{\lambda} r^{\lambda}-C_{-\beta-1} r^{-\beta-1} \Theta(1-r)\right) d r, \\
\text { если }-\beta-1 \in J .
\end{array}\right.
$$

Отметим, что при $-\beta-1 \notin J$ функция $r_{J}^{\beta}$ однородна степени $\beta$. Действительно,

$$
\begin{gathered}
\left((k r)_{J}^{\beta}, \varphi(r)\right)=\frac{1}{k}\left(r_{J}^{\beta}, \varphi\left(\frac{r}{k}\right)\right)=\int_{0}^{\infty} r^{\beta}\left(\varphi\left(\frac{r}{k}\right)-\sum_{\substack{\lambda \in J \\
\lambda<-\beta-1}} \frac{C_{\lambda}}{k^{\lambda}} r^{\lambda}\right) d r \\
=k^{\beta} \int_{0}^{\infty} r^{\beta}\left(\varphi(r)-\sum_{\substack{\lambda \in J \\
\lambda<-\beta-1}} C_{\lambda} r^{\lambda}\right) d r .
\end{gathered}
$$

При $-\beta-1 \in J$ обобщенная функция $r_{J}^{\beta}$ не является однородной.

Заметим, что при $J=\mathbb{Z}_{+}=\{0,1,2, \ldots\}$ пространство $\mathscr{S}_{J}=\mathscr{S}_{+}$, a $r_{J}^{\beta}=r_{+}^{\beta}$ и $\triangle_{\lambda}(r)=\frac{(-1)^{\lambda}}{\lambda !} \delta^{(\lambda)}(r)($ cм. [6]).

Лемма 2.1. Если $F(r) \in \mathscr{S}_{J}^{\prime}$ имеет компактный носитель, то на $\mathscr{S}_{J}$ справедливо следующее асимптотическое разложение

$$
F(k r) \sim \sum_{\lambda \in J} \frac{1}{k^{\lambda+1}} \triangle_{\lambda}(r) M_{\lambda}[F], \quad k \rightarrow+\infty
$$

т.е. для любого $N$ и любой основной функиии $\psi(r) \in \mathscr{S}_{J}$

$$
(F(k r), \psi(r))=\sum_{\substack{\lambda \in J \\ \lambda \leqslant N}} \frac{1}{k^{\lambda+1}}\left(\Delta_{\lambda}(r), \psi(r)\right) M_{\lambda}[F]+o_{\psi}\left(\frac{1}{k^{N+1}}\right), \quad k \rightarrow+\infty
$$

ДоказАтельство. Пусть функция $\xi(r) \in C^{\infty}\left(\overline{\mathbb{R}}_{+}\right)$, финитна и $\xi(r)=1$ в окрестности носителя $F(r)$, а $N$ - фиксированное число. Для любого $M$ 
и любой $\psi(r) \in \mathscr{S}_{J}$ имеем

$$
\begin{gathered}
(F(k r), \psi(r))=\frac{1}{k}\left(F(r), \psi\left(\frac{r}{k}\right)\right)=\frac{1}{k}\left(F(r), \xi(r) \psi\left(\frac{r}{k}\right)\right) \\
=\frac{1}{k}\left(F(r), \xi(r)\left[\psi\left(\frac{r}{k}\right)-\sum_{\substack{\lambda \in J \\
\lambda \leqslant M}} \frac{C_{\lambda}}{k^{\lambda}} r^{\lambda}\right]\right)+\frac{1}{k}\left(F(r), \xi(r) \sum_{\substack{\lambda \in J \\
\lambda \leqslant M}} \frac{C_{\lambda}}{k^{\lambda}} r^{\lambda}\right) \\
=\frac{1}{k}\left(F(r), \xi(r) \omega\left(\frac{r}{k}\right)\right)+\sum_{\substack{\lambda \in J \\
\lambda \leqslant M}} \frac{1}{k^{\lambda+1}}\left(\Delta_{\lambda}(r), \psi(r)\right) M_{\lambda}[F],
\end{gathered}
$$

где $\omega(r)=\psi(r)-\sum_{\substack{\lambda \in J \\ \lambda \leqslant M}} C_{\lambda} r^{\lambda}$. В силу конечности порядка функционала $F(r)$ существуют числа $Q$ и $c_{1}$ такие, что

$$
\left|\frac{1}{k}\left(F(r), \xi(r) \omega\left(\frac{r}{k}\right)\right)\right| \leqslant c_{1} \mathscr{P}_{Q}\left(\xi(r) \omega\left(\frac{r}{k}\right)\right)=o\left(\frac{1}{k^{N+1}}\right),
$$

если $M$ достаточно велико. Лемма доказана.

СлеДСТВИЕ. Пусть $\rho(k)$ - автомодельная функиия порядка $\beta$, a $F(r) \in \mathscr{S}_{J}^{\prime}$ имеет компактный носитель и для любого $\lambda \in J, \lambda \leqslant-\beta-1$,

$$
M_{\lambda}[F]=0 .
$$

Тогда $F(r)$ имеет тривиальную квазиасимптотику относительно $\rho(k)$.

Это утверждение непосредственно следует из оценок леммы 0.1.

Пусть $F(r) \in \mathscr{S}_{J}^{\prime}, \nu>0, \rho(k)$ - автомодельная функция порядка $\beta$. Положим $F_{\nu}(r)=r^{\nu} F(r)$. Ясно, что $F_{\nu} \in \mathscr{S}_{J-\nu}^{\prime}$, где $J-\nu=\{\lambda-\nu, \lambda \in J\}$. Если $F \in A O_{\rho}\left(\mathscr{S}_{J}\right)$, то $F_{\nu} \in A O_{\rho_{\nu}}\left(\mathscr{S}_{J-\nu}\right)$, где $\rho_{\nu}(k)=k^{\nu} \rho(k)$ имеет порядок $\beta+\nu$. Это непосредственно следует из соотношения

$$
\frac{1}{\rho_{\nu}(k)}\left(F_{\nu}(k r), \varphi(r)\right)=\frac{1}{\rho(k)}\left(F(k r), r^{\nu} \varphi(r)\right) .
$$

Следующие леммы и теоремы дают описание обобщенных функций из класса $A O_{\rho}\left(\mathscr{S}_{J}\right)$.

Лемма 2.2. Пусть $J$ - допустимое множество вещественных чисел $u$ $\rho(k)$ - автомодельная функиия порядка $\beta$, причем $-\beta-1 \notin J$. Пусть $A, \ell \in \mathbb{R}$, $N \in \mathbb{Z}_{+}$и $\gamma(r)$ - непрерьвная при $r>0$ функция такая, что

$$
\gamma(r) \sim A \rho(r) r^{N+\ell}, \quad r \rightarrow+\infty .
$$

Определим обобщенную функцию $F_{1}(r) \in \mathscr{S}_{J}^{\prime}$ формулой

$$
\left(F_{1}(r), \psi(r)\right)=\int_{1}^{\infty} \gamma(r)\left(\frac{d}{d r}\right)^{N}\left(r^{-\ell}\left(\psi(r)-\sum_{\substack{\lambda \in J \\ \lambda<-\beta-1}} C_{\lambda} r^{\lambda}\right)\right) d r \quad \forall \psi \in \mathscr{S}_{J}
$$

Тогда $F_{1}(r) \in A O_{\rho}\left(\mathscr{S}_{J}\right)$, причем

$$
\frac{1}{\rho(k)} F_{1}(k r) \underset{k \rightarrow \infty}{\longrightarrow}(-1)^{N} N !\left(\begin{array}{c}
\beta+\ell+N \\
N
\end{array}\right) A r_{J}^{\beta} .
$$


ДокАЗАТЕЛЬСтво. Положим

$$
g(r)=\left(\frac{d}{d r}\right)^{N}\left(\frac{1}{r^{\ell}}\left(\psi(r)-\sum_{\substack{\lambda \in J \\ \lambda<-\beta-1}} C_{\lambda} r^{\lambda}\right)\right) .
$$

Имеют место следующие оценки:

$$
|g(r)| \leqslant c \begin{cases}r^{-\beta-1-\Delta-\ell-N} & \text { для } r \text { в окрестности бесконечности, } \\ r^{-\beta-1+\Delta_{1}-\ell-N} & \text { для } r \text { в окрестности нуля, }\end{cases}
$$

где $\Delta, \Delta_{1}>0$. Для получения этой оценки мы воспользовались тем что последнее $\lambda$ в сумме равно $-\beta-1-\triangle$, а в окрестности нуля

$$
\psi(r)-\sum_{\substack{\lambda \in J \\ \lambda<-\beta-1}} C_{\lambda} r^{\lambda} \sim \text { const } \cdot r^{\lambda_{1}}, \quad \lambda_{1} \in J, \quad \lambda_{1}=-\beta-1+\Delta_{1}, \quad \Delta_{1}>0 .
$$

Кроме того,

$$
|\gamma(r)| \leqslant \text { const } \cdot \rho(r) r^{N+\ell} \leqslant c_{\varepsilon} r^{N+\ell+\beta+\varepsilon}, \quad r>1,
$$

где $\varepsilon<\Delta$. Отсюда, в частности, следует, что интеграл в (2.6) сходится.

Покажем, что $F_{1}(r)$, определяемая формулой $(2.6)$, обладает квазиасимптотикой на бесконечности относительно $\rho(k)$. Для любой $\psi(r) \in \mathscr{S}_{J}$ имеем

$$
\begin{aligned}
\frac{1}{\rho(k)} & \left(F_{1}(k r), \psi(r)\right)=\frac{1}{k \rho(k)}\left(F_{1}(r), \psi\left(\frac{r}{k}\right)\right) \\
& =\frac{1}{k \rho(k)} \int_{1}^{\infty} \gamma(r)\left(\frac{d}{d r}\right)^{N} \frac{\psi(r / k)-\sum_{\substack{\lambda \in J \\
\lambda<-\beta-1}} C_{\lambda} r^{\lambda} / k^{\lambda}}{r^{\ell}} d r \\
& =\frac{1}{k^{N+\ell} \rho(k)} \int_{1 / k}^{\infty} \gamma(k r)\left(\frac{d}{d r}\right)^{N} \frac{\psi(r)-\sum_{\substack{\lambda \in J \\
\lambda<-\beta-1}} C_{\lambda} r^{\lambda}}{r^{\ell}} d r \\
& =\int_{1 / k}^{\infty} \frac{\rho(k r)}{\rho(k)} \frac{\gamma(k r)}{(k r)^{N+\ell} \rho(k r)} r^{N+\ell} g(r) d r .
\end{aligned}
$$

Полагая, что $\varepsilon<\min \left\{\Delta, \Delta_{1}\right\}$, по лемме 0.1 имеем

$$
\frac{\rho(k r)}{\rho(k)} \leqslant c \begin{cases}r^{\beta+\varepsilon} & \text { при } r>1, k>1, \\ r^{\beta-\varepsilon} & \text { при } \frac{1}{k}<r<1, k>1 .\end{cases}
$$

Из оценок (2.8), (2.10) и оценки

$$
\left|\frac{\gamma(k r)}{(k r)^{N+\ell} \rho(k r)}\right| \leqslant \text { const }
$$

справедливой при $k r>1$, следует, что подынтегральная функция в (2.9) оценивается в окрестности бесконечности функцией $c r^{-1-(\Delta-\varepsilon)}$, а в окрестности нуля - функцией $c r^{-1+\left(\Delta_{1}-\varepsilon\right)}$, т.е. интегрируемой функцией, не зависящей от $k$. 
При $k \rightarrow+\infty$ у подынтегральной функции при каждом $r$ существует предел. По теореме Лебега в (2.9) можно перейти к пределу при $k \rightarrow+\infty$. Имеем (“избавляясь" в пределе от производных) соотношение

$$
\frac{1}{\rho(k)}\left(F_{1}(k r), \psi(r)\right) \underset{k \rightarrow+\infty}{\longrightarrow}(-1)^{N} A G \int_{0}^{\infty} r^{\beta}\left(\psi(r)-\sum_{\substack{\lambda \in J \\ \lambda<-\beta-1}} C_{\lambda} r^{\lambda}\right) d r,
$$

где

$$
G=(\beta+\ell+N)(\beta+\ell+N-1) \cdots(\beta+\ell+1)
$$

Лемма доказана.

ЗАмЕчАниЕ. Утверждение леммы останется справедливым и в том случае, когда $-\beta-1 \in J$ и $-\beta-1-\ell \in \mathbb{Z}_{+}$, а $N>-\beta-1-\ell$. При этом вместо (2.7) имеет место соотношение

$$
\frac{1}{\rho(k)} F_{1}(k r) \underset{k \rightarrow+\infty}{\longrightarrow}(-1)^{\beta+\ell+N+1} A(\beta+\ell+N) !(-\beta-\ell-1) ! \Delta_{-\beta-1}(r) .
$$

Действительно, оценка (2.8) остается справедливой и в этом случае, так как

$$
\left(\frac{d}{d r}\right)^{N}\left(r^{-\ell} C_{-\beta-1} r^{-\beta-1}\right)=0 .
$$

Переходя к пределу в (2.9), получим

$$
\begin{gathered}
\frac{1}{\rho(k)}\left(F_{1}(k r), \psi(r)\right) \underset{k \rightarrow+\infty}{\longrightarrow} A \int_{0}^{\infty} r^{\beta+\ell+N}\left(\frac{d}{d r}\right)^{N}\left[r^{-\ell}\left(\psi(r)-\sum_{\substack{\lambda \in J \\
\lambda<-\beta-1}} C_{\lambda} r^{\lambda}\right)\right] d r \\
=A \int_{0}^{\infty} r^{\beta+\ell+N}\left(\frac{d}{d r}\right)^{N}\left[r^{-\ell}\left(\psi(r)-\sum_{\substack{\lambda \in J \\
\lambda<-\beta-1}} C_{\lambda} r^{\lambda}-C_{-\beta-1} r^{-\beta-1} \eta(r)\right)\right] d r \\
+A C_{-\beta-1} \int_{0}^{\infty} r^{\beta+\ell+N}\left(\frac{d}{d r}\right)^{N} r^{-\ell-\beta-1} \eta(r) d r
\end{gathered}
$$

Нетрудно видеть (интегрируя по частям $\ell+\beta+N+1$ раз), что первое слагаемое в последней сумме равно нулю. Интегрируя по частям нужное число раз второе слагаемое и учитывая, что $\eta(0)=1$, доказываем соотношение (2.14).

Лемма 2.3. Пусть $F(r) \in \mathscr{S}_{J}^{\prime}$, причем $F(r) \in A O_{\rho}\left(\mathscr{S}_{J}\right)$, где $\rho(k)$ - автомодельная функиия порядка $\beta$. Тогда существуют $F_{0}(r) \in \mathscr{S}_{J}^{\prime}$ с компактным носителем и моментами, удовлетворяющими условиям (2.4), целые числа $N \geqslant 0$ и $\ell \geqslant 0$, непрерьвная функиия $\gamma(r)$, имеющая свойства (2.5), такие, что справедлива формула

$$
F(r)=F_{0}(r)+F_{1}(r)
$$

где $F_{1}$ определяется формулой (2.6). 
ДокАЗАТЕЛЬство. Прежде всего заметим, что существует обобщенная функция $F_{0}(r) \in \mathscr{S}_{J}^{\prime}$ с компактным по $r$ носителем такая, что

$$
F_{0}(r)=F(r), \quad r<1, \quad M_{\lambda}\left[F_{0}\right]=0, \quad \lambda \in J, \quad \lambda \leqslant-\beta-1
$$

(см., например, далее доказательство леммы 3.1). Следовательно, согласно следствию к лемме 2.1 обобщенная функция $F_{0}$ обладает тривиальной квазиасимптотикой относительно $\rho(k)$. Положим $F(r)=F_{1}(r)+F_{0}(r)$. Тогда $F_{1}(r) \in A O_{\rho}\left(\mathscr{S}_{J}\right)$ и $\operatorname{supp} F_{1}(r) \subset\{r \geqslant 1\}$. Докажем, что $F_{1}(r)$ представляется в виде $(2.6)$.

Ясно, что $F_{1} \in \mathscr{S}_{+}^{\prime}$ и $F_{1} \in A O_{\rho}\left(\mathscr{S}_{J_{\varnothing}}\right)$. Поэтому в силу теоремы о сходимости в конечном порядке (см. [7]) существует $\ell \in \mathbb{Z}_{+}$такое, что $F_{1} \in A O_{\rho}\left(\mathscr{S}_{+, \ell}\right)$, где $\mathscr{S}_{+, \ell}=\left\{\varphi \in \mathscr{S}_{+}: \varphi(r)=r^{\ell} \psi(r), \psi(r) \in \mathscr{S}_{+}\right\}$. Тогда обобщенная функция $r^{\ell} F_{1}(r)$ обладает квазиасимптотикой относительно $k^{\ell} \rho(k)$ на всем $\mathscr{S}_{+}$, и поэтому существует такое число $N$, что ее первообразная порядка $N$ - непрерывная функция, имеющая асимптотику относительно $r^{\ell+N} \rho(r)$ (см. (0.8)), т.е.

$$
\gamma(r)=(-1)^{N}\left(r^{\ell} F_{1}(r)\right)^{(-N)} \sim \text { const } \cdot r^{\ell+N} \rho(r), \quad r \rightarrow+\infty .
$$

Полагая $\eta_{\varkappa}(r)=\eta(r / \varkappa)$, где $\eta(r)$ удовлетворяет условиям (1.5), для любого $\varkappa>0$ и любой $\psi(r) \in \mathscr{S}_{J}$ имеем

$$
\left(F_{1}(r), \psi(r)\right)=\left(F_{1}(r), \psi(r)-\sum_{\substack{\lambda \in J \\ \lambda<-\beta-1}} C_{\lambda} r^{\lambda} \eta_{\varkappa}(r)\right)+\sum_{\substack{\lambda \in J \\ \lambda<-\beta-1}} C_{\lambda}\left(F_{1}(r), r^{\lambda} \eta_{\varkappa}(r)\right) .
$$

Покажем, что второе слагаемое справа в (2.17) стремится к нулю при $\varkappa \rightarrow+\infty$. Действительно, учитывая, что $r^{\lambda} \eta(r) \in \mathscr{S}_{J}, \lambda \in J$, и $F_{1} \in A O_{\rho}\left(\mathscr{S}_{J}\right)$ (и поэтому $\left.\left(F_{1}(\varkappa r), r^{\lambda} \eta(r)\right) \sim c \rho(\varkappa)\right)$, а также то, что $\lambda+\beta+1<0$, имеем для любого слагаемого этой суммы

$$
\left(F_{1}(r), r^{\lambda} \eta_{\varkappa}(r)\right)=\varkappa^{1+\lambda}\left(F_{1}(\varkappa r), r^{\lambda} \eta(r)\right) \sim c \varkappa^{1+\lambda} \rho(\varkappa) \underset{\varkappa \rightarrow+\infty}{\longrightarrow} 0 .
$$

Для первого слагаемого (2.17) имеем

$$
\begin{aligned}
& \left(F_{1}(r), \psi(r)-\sum_{\substack{\lambda \in J \\
\lambda<-\beta-1}} C_{\lambda} r^{\lambda} \eta_{\varkappa}(r)\right) \\
& =(-1)^{N}\left(\left(r^{\ell} F_{1}(r)\right)^{(-N)},\left(\frac{d}{d r}\right)^{N}\left(r^{-\ell}\left(\psi(r)-\sum_{\substack{\lambda \in J \\
\lambda<-\beta-1}} C_{\lambda} r^{\lambda} \eta_{\varkappa}(r)\right)\right)\right. \\
& =\int_{1}^{\infty} \gamma(r)\left(\frac{d}{d r}\right)^{N}\left(r^{-\ell}\left(\psi(r)-\sum_{\substack{\lambda \in J \\
\lambda<-\beta-1}} C_{\lambda} r^{\lambda} \eta_{\varkappa}(r)\right)\right) d r
\end{aligned}
$$

Учитывая то, что выражение слева в (2.17) не зависит от $\varkappa$, а второе слагаемое справа стремится к нулю при $\varkappa \rightarrow+\infty$, выводим, что и первое слагаемое справа 
имеет предел. Покажем, что оно стремится к интегралу в формуле (2.6). Для этого рассмотрим каждое слагаемое в сумме (2.18) под знаком интеграла. По формуле Лейбница имеем

$$
\left(\frac{d}{d r}\right)^{N} r^{\lambda-\ell} \eta_{\varkappa}(r)=\sum_{m=0}^{N} C_{m} r^{\lambda-\ell-(N-m)} \frac{1}{\varkappa^{m}} \eta^{(m)}\left(\frac{r}{\varkappa}\right) .
$$

Теперь для каждого слагаемого этой суммы, учитывая (1.5), имеем при $m \neq 0$

$$
\begin{aligned}
& \left|\int_{1}^{\infty} \gamma(r) r^{\lambda-\ell-N+m} \frac{1}{\varkappa^{m}} \eta^{(m)}\left(\frac{r}{\varkappa}\right) d r\right| \leqslant \frac{c}{\varkappa^{m}} \int_{\varkappa}^{2 \varkappa} r^{\lambda-\ell-N+m} r^{N+\ell} \rho(r) d r \\
& \quad \leqslant \frac{c_{1}}{\varkappa^{m}} \int_{\varkappa}^{2 \varkappa} r^{-\beta-1-\Delta+m} r^{\beta+\varepsilon} d r \leqslant \frac{c_{2}}{\varkappa^{m}} \int_{\varkappa}^{2 \varkappa} r^{-1+m-(\Delta-\varepsilon)} d r \\
& \quad \leqslant \frac{c_{3}}{\varkappa^{\Delta-\varepsilon}} \underset{\varkappa \rightarrow+\infty}{\longrightarrow} 0 .
\end{aligned}
$$

Здесь мы учли, что $\lambda=-\beta-1-\Delta, \Delta>0$, асимптотическое соотношение $(2.16)$, свойства автомодельной функции и воспользовались леммой 0.1 при $\varepsilon<\Delta$. Учитывая, что $\eta_{\varkappa}(r) \rightarrow 1$ при каждом фиксированном $r$, для слагаемого с индексом $m=0$ имеем

$$
\int_{1}^{2 \varkappa} r^{\lambda-\ell-N} \gamma(r) \eta\left(\frac{r}{\varkappa}\right) d r \underset{\varkappa \rightarrow+\infty}{\longrightarrow} \int_{1}^{\infty} r^{\lambda-\ell-N} \gamma(r) d r .
$$

Переходя теперь в $(2.17)$ к пределу при $\varkappa \rightarrow+\infty$, получим требуемое утверждение. Лемма доказана.

ЗАмЕчАниЕ. Из доказательства леммы видно, что представление (2.6) для $F_{1}(r)$ останется справедливым для тех $\ell$, для которых функция $r^{\ell} F_{1}(r)$ асимптотически однородна относительно $k^{\ell} \rho(k)$ на $\mathscr{S}_{+}$. В частности, если сама $F_{1}(r) \in A O_{\rho}\left(\mathscr{S}_{+}\right)$, то в $(2.5)-(2.7)$ можно считать $\ell=0$.

УТВЕРЖДЕНИЕ 2.1. Пусть $J$ и $J_{1}$ - допустимые множества вещественных чисел, $J \subset J_{1}$ и $\rho(k)$ - автомодельная функиия порядка $\beta$. Пусть $F(r) \in \mathscr{S}_{J}^{\prime}$ и $F(r) \in A O_{\rho}\left(\mathscr{S}_{J}\right)$. Тогда справедливы следующие утверждения.

1) Если $-\beta-1 \notin J_{1}$, то $F(r)$ продолжсается на $\mathscr{S}_{J_{1}}$ до $\widehat{F}(r) \in A O_{\rho}\left(\mathscr{S}_{J_{1}}\right)$ так, что на $\mathscr{S}_{J_{1}}$

$$
\frac{1}{\rho(k)} \widehat{F}(k r) \underset{\rightarrow \infty}{\longrightarrow} B r_{J_{1}}^{\beta}
$$

для некоторого $B \in \mathbb{R}$. Любое другое такое продолжение отличается от $\widehat{F}(r)$ на

$$
\sum_{\substack{\lambda \in J_{1} \backslash J \\-\beta-1<\lambda \leqslant M}} a_{\lambda} \Delta_{\lambda}(r)
$$

с некоторыми постоянными $a_{\lambda} u M$.

2) $Е с л и-\beta-1 \notin J u-\beta-1 \in J_{1}$, то $F(r)$ продолжается на $\mathscr{S}_{J_{1}}$ до $\widehat{F}(r) \in A O_{\widehat{\rho}}\left(\mathscr{S}_{J_{1}}\right)$. При этом на $\mathscr{S}_{J_{1}}$ имеет место соотношение

$$
\frac{1}{\widehat{\rho}(k)} \widehat{F}(k r) \underset{\rightarrow \infty}{\longrightarrow} \pm B \triangle_{-\beta-1}(r),
$$


где выбирается знак “+”, если $\int_{1}^{\infty} \frac{\rho(\varkappa) d \varkappa}{\varkappa^{\beta+1}}=\infty$, и выбирается знак "-" - если $\int_{1}^{\infty} \frac{\rho(\varkappa) d \varkappa}{\varkappa^{\beta+1}}<\infty$, число В берется из соотношения (2.19) с $J_{1}=J$.

3) Если $-\beta-1 \in J$, то $F(r)$ продолжается на $\mathscr{S}_{J_{1}}$ до $\widehat{F}(r) \in A O_{\rho}\left(\mathscr{S}_{J_{1}}\right)$. При этом на $\mathscr{S}_{J_{1}}$ имеет место соотношение

$$
\frac{1}{\rho(k)} \widehat{F}(k r) \underset{k \rightarrow \infty}{\longrightarrow} B \triangle_{-\beta-1}(r)
$$

с некоторым числом $B$.

ДокАЗАтельство. Рассмотрим случай 1$)$. По лемме $2.3 F(r)$ представляется в виде $F=F_{0}+F_{1}$, где $F_{0}$ имеет компактный носитель и $M_{\lambda}\left[F_{0}\right]=0, \lambda \in J$, $\lambda<-\beta-1$ (см. (2.4)), а $F_{1}$ представляется в виде $(2.4),(2.5)$. Продолжим $F_{0}$ до обобщенной функции $\widehat{F}_{0} \in \mathscr{S}_{J_{1}}^{\prime}$ так, чтобы

$$
\left(\widehat{F}_{0}(r), r^{\lambda}\right)=0, \quad \lambda \subset J_{1} \backslash J, \quad \lambda<-\beta-1 .
$$

Это возможно, так как если $\widehat{G}_{0}(r)$ - какое-то ее продолжение на $\mathscr{S}_{J_{1}}$, то можно положить

$$
\widehat{F}_{0}(r)=\widehat{G}_{0}(r)-\sum_{\substack{\lambda \subset J_{1} \backslash J \\ \lambda<-\beta-1}} M_{\lambda}\left[\widehat{G}_{0}\right] \triangle_{\lambda}(r) .
$$

Ясно, что $M_{\lambda}\left[\widehat{F}_{0}\right]=0$ для $\lambda \in J_{1}, \lambda<-\beta-1$. Поэтому $\frac{1}{\rho(k)} \widehat{F}_{0}(k r) \rightarrow 0, k \rightarrow \infty$, в $\mathscr{S}_{J_{1}}$. Теперь зададим $\widehat{F}_{1}$ формулой

$$
\left(\widehat{F}_{1}(r), \psi(r)\right)=\int_{1}^{\infty} \gamma(r)\left(\frac{d}{d r}\right)^{N}\left(r^{-\ell}\left(\psi(r)-\sum_{\substack{\lambda \in J_{1} \\ \lambda<-\beta-1}} C_{\lambda} r^{\lambda}\right)\right) d r, \quad \psi \in \mathscr{S}_{J_{1}}
$$

где $\gamma(r), \ell, N$ берутся из представления $(2.5)-(2.6)$ для $F(r)$. По лемме 2.2 $\widehat{F}_{1} \in A O_{\rho}\left(\mathscr{S}_{J_{1}}\right)$. Таким образом, $\widehat{F}=\widehat{F}_{1}+\widehat{F}_{0}$ дает нужное продолжение. Соотношение (2.19) следует из леммы 2.2.

Теперь поскольку все такие продолжения совпадают на основных функциях из $\mathscr{S}_{J}$, то все они отличаются друг от друга только в нуле. Поэтому если $\widehat{G}-$ другое такое продолжение, то

$$
\widehat{G}(r)-\widehat{F}(r)=\sum_{\substack{\lambda \in J_{1} \backslash J \\ \lambda \leqslant M}} a_{\lambda} \Delta_{\lambda}(r)
$$

с некоторыми постоянными $a_{\lambda}$ и $M$.

Так как оба продолжения из $A O_{\rho}\left(\mathscr{S}_{J_{1}}\right)$, то в $\mathscr{S}_{J_{1}}^{\prime}$ имеем

$$
\frac{1}{\rho(k)}(\widehat{G}(k r)-\widehat{F}(k r))=\frac{1}{\rho(k)} \sum_{\substack{\lambda \in J_{1} \backslash J \\ \lambda \leqslant M}} a_{\lambda} \Delta_{\lambda}(k r)=\sum_{\substack{\lambda \in J_{1} \backslash J \\ \lambda \leqslant M}} \frac{a_{\lambda}}{\rho(k) k^{\lambda+1}} \Delta_{\lambda}(r) \underset{k \rightarrow \infty}{\longrightarrow} g(r) .
$$

Но это возможно, только если члены в сумме с $\lambda+1+\beta>0$ отсутствуют. Отсюда получаем (2.20). 
Рассмотрим случай 2). Положим $J_{1}^{0}=J_{1} \backslash\{-\beta-1\}$. Тогда по уже доказанному $F(r)$ продолжается на $\mathscr{S}_{J_{1}^{0}}$ до $\widetilde{F}(r) \in A O_{\rho}\left(\mathscr{S}_{J_{1}^{0}}\right)$. Согласно лемме 2.3 справедливо соотношение $\widetilde{F}=\widetilde{F}_{0}+\widetilde{F}_{1}$. При этом $\widetilde{F}_{0}$ имеет много моментов, равных нулю (считаем, что $M_{\lambda}\left[\widetilde{F}_{0}\right]=0$ при $\lambda \in J_{1}^{0}, \lambda \leqslant-\beta-1$ ), а $\widetilde{F}_{1}$ удовлетворяет условиям (2.5)-(2.7), где $J$ надо заменить на $J_{1}^{0}$. Теперь $\widetilde{F}_{0}$ продолжается до $\widehat{F}_{0}$ на все $\mathscr{S}_{J_{1}}$ с тривиальной квазиасимптотикой относительно $\rho(k)$, а следовательно, и относительно $\widehat{\rho}(k)$, см. (0.6).

Для того чтобы продолжить $\widetilde{F}_{1}$ до $\widehat{F}_{1}$ на $\mathscr{S}_{J_{1}}$, достаточно определить это продолжение на основной функции $r^{-\beta-1} \eta(r)$ с функцией $\eta(r)$, удовлетворяющей условию (1.5). Положим

$$
\begin{aligned}
\left(\widehat{F}_{1}(r), r^{-\beta-1} \eta(r)\right)=D=\int_{1}^{\infty} \gamma(r)\left(\frac{d}{d r}\right)^{N}\left(r^{-\ell-\beta-1} \eta(r)\right) d r & \text { если } \int_{1}^{\infty} \frac{\rho(r) d r}{r^{\beta+1}}=\infty \\
& -\left\{\begin{array}{lr}
0, & \text { если } \int_{1}^{\infty} \frac{\rho(r) d r}{r^{\beta+1}}<\infty \\
(-1)^{N} \frac{(\beta+\ell+N) !}{(\beta+\ell) !} \int_{1}^{\infty} \frac{\gamma(r) d r}{r^{N+\beta+\ell+1}},
\end{array}\right.
\end{aligned}
$$

Здесь $\gamma(r)$ берется из представления (2.5)-(2.6) для $\widetilde{F}_{1} \in \mathscr{S}_{J_{1}^{0}}^{\prime}$.

Теперь для любой $\varphi(r) \in \mathscr{S}_{J_{1}}$ имеем

$$
\begin{aligned}
\left(\widehat{F}_{1}(k r), \varphi(r)\right)=\frac{1}{k}\left(\widehat{F}_{1}(r), \varphi\left(\frac{r}{k}\right)\right) \\
=\frac{1}{k}\left(\widetilde{F}_{1}(r), \varphi\left(\frac{r}{k}\right)-C_{-\beta-1}\left(\frac{r}{k}\right)^{-\beta-1} \eta(r)\right)+\frac{C_{-\beta-1}}{k^{-\beta}} D \\
=\frac{1}{k} \int_{1}^{\infty} \gamma(r)\left(\frac{d}{d r}\right)^{N}\left[r^{-\ell}\left(\varphi\left(\frac{r}{k}\right)-C_{-\beta-1}\left(\frac{r}{k}\right)^{-\beta-1} \eta(r)-\sum_{\substack{\lambda \in J_{1}^{0} \\
\lambda<-\beta-1}} \frac{C_{\lambda}}{k^{\lambda}} r^{\lambda}\right)\right] d r \\
\quad+\frac{C_{-\beta-1}}{k^{-\beta}} D .
\end{aligned}
$$

Мы учли, что $\varphi(r / k)-C_{-\beta-1}(r / k)^{-\beta-1} \eta(r) \in \mathscr{S}_{J_{1}^{0}}$, и воспользовались представлением (2.6) для $\widetilde{F}_{1}$. Далее имеем

$$
\begin{aligned}
&\left(\widehat{F}_{1}(k r), \varphi(r)\right)=\frac{1}{k} \int_{1}^{\infty} \gamma(r)\left(\frac{d}{d r}\right)^{N} \\
& \times {\left[r^{-\ell}\left(\varphi\left(\frac{r}{k}\right)-\sum_{\substack{\lambda \in J_{1}^{0} \\
\lambda<-\beta-1}} \frac{C_{\lambda}}{k^{\lambda}} r^{\lambda}-C_{-\beta-1}\left(\frac{r}{k}\right)^{-\beta-1} \eta\left(\frac{r}{k}\right)\right)\right] d r } \\
&+C_{-\beta-1} k^{\beta}\left[D+\int_{1}^{\infty} \gamma(r)\left(\frac{d}{d r}\right)^{N}\left(r^{-\ell-\beta-1}\left(\eta\left(\frac{r}{k}\right)-\eta(r)\right)\right) d r\right] .
\end{aligned}
$$

Первое слагаемое справа в (2.24) ведет себя как $\rho(k)$ (доказательство почти дословно повторяет доказательство леммы 2.2) и, следовательно, слабее чем $\widehat{\rho}(k)$. 
Покажем, что второе слагаемое $(2.24)$ ведет себя как $\widehat{\rho}(k)$. Для этого заметим, что

$$
\begin{gathered}
\int_{1}^{\infty} \gamma(r)\left(\frac{d}{d r}\right)^{N}\left(r^{-\ell-\beta-1} \eta(r)\right) d r=D_{1}<\infty, \\
\int_{1}^{\infty} \gamma(r)\left(\frac{d}{d r}\right)^{N}\left(r^{-\ell-\beta-1} \eta\left(\frac{r}{k}\right)\right) d r=\int_{1 / k}^{2} \frac{\gamma(k r)}{k^{\ell+\beta+N}}\left(\frac{d}{d r}\right)^{N}\left(r^{-\ell-\beta-1} \eta(r)\right) d r \\
=\int_{1 / k}^{1} \frac{\gamma(k r)}{k^{\ell+\beta+N}}\left(\frac{d}{d r}\right)^{N}\left(r^{-\ell-\beta-1}\right) d r+O\left(k^{-\beta} \rho(k)\right) .
\end{gathered}
$$

Первое соотношение очевидно, так как $\eta(r)=0$ при $r>2$.

Докажем второе соотношение (2.25). Действительно, пользуясь правилом Лейбница и учитывая соотношения

$$
\gamma(r) \sim A r^{N+\ell} \rho(r), \quad \frac{\rho(k r)}{\rho(k)} \sim r^{\beta},
$$

имеем при $1<r<2$

$$
\begin{aligned}
\int_{1}^{2} \frac{\gamma(k r)}{k^{\ell+\beta+N}}\left(\frac{d}{d r}\right)^{N}\left(r^{-\ell-\beta-1} \eta(r)\right) d r \\
\quad=\frac{\rho(k)}{k^{\beta}} \sum_{m=0}^{N} C_{m} \int_{1}^{2} \frac{\gamma(k r)}{(k r)^{N+\ell} \rho(k r)} \frac{\rho(k r)}{\rho(k)} \frac{\eta^{(m)}(r) d r}{r^{\beta+1-m}} \\
\quad \sim \frac{\rho(k)}{k^{\beta}} \sum_{m=0}^{N} C_{m} A \int_{1}^{2} \frac{\eta^{(m)}(r) d r}{r^{1-m}}
\end{aligned}
$$

где $C_{m}$ зависит от $\beta, \ell, N$.

Теперь, учитывая, что $\eta^{(m)}(r)=0$ для $m>0$ при $1 / k<r<1$, преобразуем оставшийся интеграл из (2.25) к виду

$$
\begin{aligned}
\int_{1 / k}^{1} & \frac{\gamma(k r)}{k^{\ell+\beta+N}}\left(\frac{d}{d r}\right)^{N}\left(r^{-\ell-\beta-1}\right) d r=\frac{(-1)^{N}}{k^{\beta}} \frac{(\beta+\ell+N) !}{(\beta+\ell) !} \int_{1 / k}^{1} \frac{\gamma(k r) d r}{(k r)^{N+\ell} r^{\beta+1}} \\
& =(-1)^{N} \frac{(\beta+\ell+N) !}{(\beta+\ell) !} \int_{1}^{k} \frac{\gamma(r)}{r^{N+\ell} \rho(r)} \frac{\rho(r) d r}{r^{\beta+1}}
\end{aligned}
$$

Отсюда следует, что если $\int_{1}^{\infty} \frac{\rho(r) d r}{r^{\beta+1}}=\infty$, то в силу (0.4) имеем

$$
\int_{1}^{k} \frac{\gamma(r)}{r^{N+\ell} \rho(r)} \frac{\rho(r) d r}{r^{\beta+1}} \sim \frac{A}{k^{\beta}} \widehat{\rho}(k)
$$

Если же $\int_{1}^{\infty} \frac{\rho(r) d r}{r^{\beta+1}}<\infty$, то, обозначая $\int_{1}^{\infty} \frac{\gamma(r) d r}{r^{N+\ell+\beta+1}}=D_{2}$, получим

$$
D_{2}-\int_{1}^{k} \frac{\gamma(r) d r}{r^{N+\ell+\beta+1}}=\int_{k}^{\infty} \frac{\gamma(r)}{r^{N+\ell} \rho(r)} \frac{\rho(r) d r}{r^{\beta+1}} \sim \frac{A}{k^{\beta}} \widehat{\rho}(k) .
$$


Теперь из (2.23), (2.25)-(2.28) следует, что второе слагаемое в (2.24) ведет себя как $\widehat{\rho}(k)$. При этом

$$
\frac{1}{\widehat{\rho}(k)}(\widehat{F}(k r), \varphi(r)) \underset{k \rightarrow \infty}{\longrightarrow}(-1)^{N} N ! A\left(\begin{array}{c}
\beta+\ell+N \\
N
\end{array}\right) A C_{-\beta-1}, \quad \varphi \in \mathscr{S}_{J_{1}} .
$$

Случай 2) полностью рассмотрен.

Рассмотрим случай 3). Пусть $J^{0}=J \backslash\{-\beta-1\}$ и $J_{1}^{0}=J_{1} \backslash\{-\beta-1\}$. Построим $\widehat{\widehat{F}}(r) \in A O_{\rho}\left(\mathscr{S}_{J_{1}^{0}}\right)$ как продолжение $F(r)$ с $\mathscr{S}_{J^{0}}$ на $\mathscr{S}_{J_{1}^{0}}$ в соответствии со случаем 2). Для того чтобы построить искомое продолжение $\widehat{\widehat{F}}$ на $\mathscr{S}_{J_{1}}$, достаточно определить его на одной функции вида $\eta(r) r^{-\beta-1}$, где $\eta(r)$ удовлетворяет условиям (1.5). Положим

$$
\left(\widehat{F}(r), r^{-\beta-1} \eta(r)\right)=\left(F(r), r^{-\beta-1} \eta(r)\right) .
$$

Поскольку $\eta(r) r^{-\beta-1} \in \mathscr{S}_{J}$, то $\widehat{F}(r) \in A O_{\rho}\left(\mathscr{S}_{J_{1}}\right)$.

Докажем теперь соотношение (2.22). Так как на $\mathscr{S}_{J_{1}}$

$$
\frac{1}{\rho(k)} \widehat{F}(k r) \underset{k \rightarrow \infty}{\longrightarrow} G(r),
$$

то по лемме $2.2 G(r)=A r_{J^{0}}^{\beta}$ на $\mathscr{S}_{J^{0}}$ с некоторым числом $A$ (см. $\left.(2.7)\right)$. Тогда по уже доказанному (случай 2)) на $\mathscr{S}_{J}$ имеем

$$
\frac{1}{\widehat{\rho}(k)} \widehat{F}(k r) \underset{k \rightarrow \infty}{\longrightarrow} \pm A \triangle_{-\beta-1}(r)
$$

(см. (2.21)). С другой стороны, на $\mathscr{S}_{J}$

$$
\frac{1}{\widehat{\rho}(k)} \widehat{F}(k r)=\frac{\rho(k)}{\widehat{\rho}(k)} \frac{1}{\rho(k)} F(k r) \underset{k \rightarrow \infty}{\longrightarrow} 0
$$

(см. (0.6)). Это возможно, если только $A=0$, т.е. $G(r)=0$ на $\mathscr{S}_{J^{0}}$ и, следовательно, и на $\mathscr{S}_{J_{1}^{0}}($ случай 1$\left.)\right)$. Теперь для любой $\varphi \in \mathscr{S}_{J_{1}}$ имеем

$$
\begin{gathered}
(G(r), \varphi(r))=\left(G(r), \varphi(r)-C_{-\beta-1} r^{-\beta-1} \eta(r)\right)+\left(G(r), C_{-\beta-1} r^{-\beta-1} \eta(r)\right) \\
=C_{-\beta-1}\left(G(r), r^{-\beta-1} \eta(r)\right)=C_{-\beta-1} B=B\left(\triangle_{-\beta-1}(r), \varphi(r)\right),
\end{gathered}
$$

что и доказывает соотношение (2.22). Утверждение доказано.

СледСтвиЕ. Если $F(r) \in \mathscr{S}_{J}^{\prime}-$ однородная функиия степени $\beta$, то при некотором $B \in \mathbb{R}$

$$
F(r)= \begin{cases}B r_{J}^{\beta}, & \text { если }-\beta-1 \notin J, \\ B \triangle_{-\beta-1}(r), & \text { если }-\beta-1 \in J .\end{cases}
$$

ДокАЗАТЕЛЬство. Пусть $F(r) \in \mathscr{S}_{J}^{\prime}-$ однородная функция степени $\beta$, и пусть $-\beta-1 \notin J$. Так как $F(k r)=k^{\beta} F(r)$, то $F(r) \in A O_{\rho}\left(\mathscr{S}_{J}\right)$, где $\rho(k)=k^{\beta}$. Поэтому в силу (2.19) имеем

$$
\frac{1}{\rho(k)} F(k r)=F(r) \underset{k \rightarrow \infty}{\longrightarrow} B r_{J}^{\beta},
$$

что и доказывает первое из соотношений (2.30). 
Пусть теперь $-\beta-1 \in J$. Так как $F(r) \in A O_{\rho}\left(\mathscr{S}_{J}\right)$, то соотношение $(2.22)$ доказывает справедливость (2.30) и в этом случае. Следствие доказано.

Лемма 2.4. Пусть $\rho(k)$ - автомодельная функиия порядка $\beta, J$ - допустимое множество, $-\beta-1 \notin J u-\beta-1 \notin \mathbb{Z}_{+}$. Для того чтобы $F(r) \in A O_{\rho}\left(\mathscr{S}_{J}\right)$, необходимо и достаточно, чтобы существовали числа $N, A$ и функция $\gamma(r)$, непрерывная при $r>0$, удовлетворяющая условию $\gamma(r) \sim A r^{N} \rho(r), r \rightarrow \infty$, такие, что для любой бункиии $\varphi \in \mathscr{S}_{J}$

$$
(F(r), \varphi(r))=\int_{1}^{\infty} \gamma(r)\left(\frac{d}{d r}\right)^{N}\left[\varphi(r)-\sum_{\substack{\lambda \in J \\ \lambda<-\beta-1}} C_{\lambda} r^{\lambda}\right] d r+\left(F_{0}(r), \varphi(r)\right),
$$

где $F_{0}(r) \in \mathscr{S}_{J}^{\prime}$, имеет компактный носитель и все моменты $M_{\lambda}\left[F_{0}\right]=0$ при $\lambda<-\beta-1, \lambda \in J$.

ДокАЗАТЕЛЬство. Согласно утверждению 2.1 (случай 1)) $F$ продолжается до $\widehat{F} \in A O_{\rho}\left(\mathscr{S}_{J \cup \mathbb{Z}_{+}}\right)$, так что $\widehat{F}$ обладает квазиасимптотикой относительно $\rho(k)$ на $\mathscr{S}_{+}$. Представим $\widehat{F}$ в виде $F_{1}+F_{0}$, где $\operatorname{supp} F_{1}$ отделен от нуля, а $F_{0}$ имеет компактный носитель и все моменты $M_{\lambda}\left[F_{0}\right]=0$ при $\lambda \in J \cup \mathbb{Z}_{+}, \lambda \leqslant-\beta-1$. Ясно, что обобщенная функция $F_{1}$ асимптотически однородна на $\mathscr{S}_{+}$, и по лемме 2.3 (см. замечание к ней) имеет место формула (2.31). Обратное утверждение следует из леммы 2.2. Лемма доказана.

Лемма 2.5. Пусть $\rho(k)$ - автомодельная функиия порядка $\beta, J$ - допустимое множество, $-\beta-1 \in J u-\beta-1 \in \mathbb{Z}_{+}$. Для того чтобъ $F(r) \in A O_{\rho}\left(\mathscr{S}_{J}\right)$, необходимо и достаточно, чтобы существовали числа $N(N>-\beta-1), A$ и функция $\gamma(r)$, непрерывная при $r>0$, удовлетворяющая условию $\gamma(r) \sim$ $A r^{N} \rho(r), r \rightarrow \infty$, такие, что для любой функиии $\varphi \in \mathscr{S}_{J}$

$$
(F(r), \varphi(r))=\int_{1}^{\infty} \gamma(r)\left(\frac{d}{d r}\right)^{N}\left[\varphi(r)-\sum_{\substack{\lambda \in J \\ \lambda<-\beta-1}} C_{\lambda} r^{\lambda}\right] d r+\left(F_{0}(r), \varphi(r)\right),
$$

где $F_{0}(r) \in \mathscr{S}_{J}^{\prime}$, имеет компактный носитель и все моменты $M_{\lambda}\left[F_{0}\right]=0$ при $\lambda \leqslant-\beta-1, \lambda \in J$.

ДокАЗАТЕЛЬСтво. Согласно утверждению 2.1 (случай 3$)$ ) $F$ продолжается до $\widehat{F} \in A O_{\rho}\left(\mathscr{S}_{J \cup \mathbb{Z}_{+}}\right)$, т.е. $\widehat{F}$ обладает квазиасимптотикой относительно $\rho(k)$ на $\mathscr{S}_{+}$. Представим $\widehat{F}$ в виде $F_{1}+F_{0}$, где $\operatorname{supp} F_{1}$ отделен от нуля, а $F_{0}$ имеет компактный носитель и все моменты $M_{\lambda}\left[F_{0}\right]=0$ при $\lambda \in J \cup \mathbb{Z}_{+}, \lambda \leqslant-\beta-1$. Ясно, что обобщенная функция $F_{1}$ асимптотически однородна на $\mathscr{S}_{+}$, и по лемме 2.3 (см. замечание к ней) имеет место формула (2.32). Обратное утверждение, с учетом того, что $N>-\beta-1$, следует из замечания к лемме 2.2 . Лемма доказана.

Теорема 2.1. Пусть $F(r) \in \mathscr{S}_{J}^{\prime}, \rho(k)$ - автомоделъная функиия порядка $\beta$, $J$ - допустимое множество, а число $\nu$ таково, что

$$
\begin{cases}-\beta-1-\nu \notin \mathbb{Z}_{+}, & \text {если }-\beta-1 \notin J \\ -\beta-1-\nu \in \mathbb{Z}_{+}, & \text {если }-\beta-1 \in J\end{cases}
$$


Тогда для того чтобы $F(r) \in A O_{\rho}\left(\mathscr{S}_{J}\right)$, необходимо и достаточно, чтобъ существовали числа $N(N>-\beta-1-\nu$ в случае $-\beta-1 \in J), A$ и функция $\gamma(r)$, непрерывная при $r>0$, имеющая асимптотику

$$
\gamma(r) \sim A r^{\nu+N} \rho(r), \quad r \rightarrow+\infty
$$

такие, что для любой основной функции $\psi \in \mathscr{S}_{J}$

$$
(F(r), \psi(r))=\int_{1}^{\infty} \gamma(r)\left(\frac{d}{d r}\right)^{N}\left[r^{-\nu}\left(\psi(r)-\sum_{\substack{\lambda \in J \\ \lambda<-\beta-1}} C_{\lambda} r^{\lambda}\right)\right] d r+\left(F_{0}(r), \psi(r)\right)
$$

где $F_{0}(r) \in \mathscr{S}_{J}^{\prime}$, имеет компактный носитель и $M_{\lambda}\left[F_{0}\right]=0$ для $\lambda \in J, \lambda \leqslant-\beta-1$.

ДокАЗАТЕЛЬСтво. Положим $F_{\nu}(r)=r^{\nu} F(r)$. Тогда $F_{\nu} \in A O_{\rho_{\nu}}\left(\mathscr{S}_{J-\nu}\right)$, где $\rho_{\nu}(k)=k^{\nu} \rho(k)$ с порядком автомодельности $\beta_{\nu}=\beta+\nu$. Рассмотрим сначала случай $-\beta-1 \notin J$. Поскольку тогда

$$
-\beta_{\nu}-1=-\beta-\nu-1 \notin \mathbb{Z}_{+}, \quad-\beta_{\nu}-1 \notin J-\nu,
$$

то по лемме 2.4 для любой $\varphi \in \mathscr{S}_{J-\nu}$ имеем

$$
\left(\widetilde{F}_{\nu}(r), \varphi(r)\right)=\int_{1}^{\infty} \gamma_{\nu}(r)\left(\frac{d}{d r}\right)^{N}\left[\varphi(r)-\sum_{\substack{\lambda \in J-\nu \\ \lambda<-\widetilde{\beta}-1}} C_{\lambda}^{\varphi} r^{\lambda}\right] d r+\left(\widetilde{F}_{0}(r), \varphi(r)\right)
$$

где $\gamma_{\nu}(r)$ - функция, непрерывная при $r>0$, и

$$
\gamma_{\nu}(r) \sim r^{N} \rho_{\nu}(r)=r^{N+\nu} \rho(r)
$$

Отсюда для любой $\psi(r) \in \mathscr{S}_{J}$ имеем

$$
\begin{aligned}
& (F(r), \psi(r))=\left(F_{\nu}(r), r^{-\nu} \psi(r)\right) \\
& =\int_{1}^{\infty} \gamma_{\nu}(r)\left(\frac{d}{d r}\right)^{N}\left[r^{-\nu} \psi(r)-\sum_{\substack{\lambda \in J-\nu \\
\lambda<-\beta-\nu-1}} C_{\lambda+\nu}^{\psi} r^{\lambda}\right] d r+\left(F_{0}(r), \psi(r)\right) \\
& \quad=\int_{1}^{\infty} \gamma_{\nu}(r)\left(\frac{d}{d r}\right)^{N}\left[\frac{\psi(r)-\sum_{\substack{\lambda \in J \\
\lambda<-\beta-1}} C_{\lambda} r^{\lambda}}{r^{\nu}}\right] d r+\left(F_{0}(r), \psi(r)\right) .
\end{aligned}
$$

Здесь мы сделали замену $\lambda^{\prime}=\lambda+\nu$, опустили штрихи и учли, что $C_{\lambda}^{\psi}=C_{\lambda-\nu}^{r^{-\nu} \psi}$, где $C_{\lambda}^{\psi}$ - коэффициенты разложения функции $\psi$ (см. (1.3), (1.4)).

Рассмотрим случай $-\beta-1 \in J$. Так как $-\beta_{\nu}-1=-\beta-\nu-1 \in \mathbb{Z}_{+}$ и $-\beta_{\nu}-1 \in J-\nu$, то по лемме 2.5 имеем соотношение (2.36). Далее, аналогично получаем (2.34). Теорема доказана. 


\section{§ 3. Асимптотически однородные обобщенные функции на пространствах $W_{J}$ и $V_{J}$}

Пусть $J$ - допустимое множество. Пространство $W_{J}$ и асимптотически однородные обобщенные функции на нем определены в 1 .

В соответствии с $(2.2)$ для любой $\Phi(e) \in \mathscr{S}^{\prime}\left(S^{n-1}\right)$ определим обобщенную функцию $r_{J}^{\beta} \otimes \Phi(e)$ формулой

$$
\begin{aligned}
& \left(r_{J}^{\beta} \otimes \Phi(e), \psi(r, e)\right) \\
& =\left\{\begin{array}{c}
\int_{0}^{\infty} r^{\beta}\left(\Phi(e), \psi(r, e)-\sum_{\substack{\lambda \in J \\
\lambda<-\beta-1}} C_{\lambda}(e) r^{\lambda}\right)_{e} d r \quad \text { при }-\beta-1 \notin J, \\
\int_{0}^{\infty} r^{\beta}\left(\Phi(e), \psi(r, e)-\sum_{\substack{\lambda \in J \\
\lambda<-\beta-1}} C_{\lambda}(e) r^{\lambda}\right. \\
\left.-C_{-\beta-1}(e) r^{-\beta-1} \Theta(1-r)\right)_{e} d r \quad \text { при }-\beta-1 \in J
\end{array}\right.
\end{aligned}
$$

для любой $\psi(r, e) \in W_{J}$.

Здесь и далее нижний индекс $е$ в выражении $\left(G_{r}(e), \psi(r, e)\right)_{e}$ означает, что рассматривается значение обобщенной функции $G_{r}(e) \in \mathscr{S}^{\prime}\left(S^{n-1}\right)$ на функции $\psi(r, e)$, рассматриваемой как основной из $\mathscr{S}\left(S^{n-1}\right)$ при фиксированном $r$.

Отметим, что при $-\beta-1 \notin J$ обобщенная функция $r_{J}^{\beta} \otimes \Phi(e)$ однородна по $r$ степени $\beta$.

Лемма 3.1. Пусть обобщенная функиия $F(r, e) \in W_{J}^{\prime}$. Тогда ее всегда можно представить в виде $F(r, e)=F_{1}(r, e)+F_{0}(r, e)$, где носитель $F_{1}$ отделен от нуля (пусть, например, $\left.\operatorname{supp} F_{1} \subset\left\{r>1, e \in S^{n-1}\right\}\right)$, a $F_{0}$ имеет компактный носитель и сколь угодно много моментов, например,

$$
M_{\lambda_{i}}\left[F_{0}\right](e) \equiv 0, \quad \lambda_{i} \in J, \quad i=1, \ldots, M .
$$

Если при этом обобщенная функция $F(r, e)$ ортогональна ко всем функциям из некоторого семейства $\mathscr{E} \in \mathscr{S}\left(S^{n-1}\right)$, m.е. $(F(r, e), \varphi(e))_{e} \equiv 0$ в $\mathscr{S}_{J}^{\prime}$ для $\varphi \in \mathscr{E}$, то такими же будут $F_{1}$ и $F_{0}$, т.е. для $\varphi \in \mathscr{E}$ в $\mathscr{S}_{J}^{\prime}$

$$
\left(F_{1}(r, e), \varphi(e)\right) \equiv 0, \quad\left(F_{0}(r, e), \varphi(e)\right) \equiv 0 .
$$

ДокАЗАТЕЛЬСтво. Пусть $\widetilde{F}(r, e)=F(r, e) \eta(r)$, где $\eta(r)$ удовлетворяет условиям $(1.5)$. Отметим, что $(\widetilde{F}(r, e), \varphi(e))_{e} \equiv 0, \varphi(e) \in \mathscr{E}$. Подберем множество бесконечно дифференцируемых функций

$$
\left\{\omega_{i}(r): \operatorname{supp} \omega_{i} \subset(1,2), \int r^{\lambda_{j}} \omega_{i}(r) d r=\delta_{i j}, i, j=1, \ldots, M, \lambda_{j} \in J\right\} .
$$

Пусть $\Omega(r, e)=\sum_{i=1}^{M} \omega_{i}(r) M_{\lambda_{i}}\left[F_{1}\right](e)$. Положим

$$
F_{1}(r, e)=F(r, e)-\widetilde{F}(r, e)+\Omega(r, e), \quad F_{0}(r, e)=\widetilde{F}(r, e)-\Omega(r, e) .
$$

Теперь легко проверить, что требуемые свойства $F_{1}(r, e)$ и $F_{0}(r, e)$ выполнены. 
Класс $A O_{\rho}\left(W_{J}\right)$ асимптотически однородных обобщенных функций относительно автомодельной функции $\rho(k)$ на пространстве $W_{J}$ определен в $\S 1$ (см. определение 1.2). Все результаты, полученные для асимптотически однородных обобщенных функций в $\S 2$ для пространства $\mathscr{S}_{J}$, практически дословно переносятся на пространство $W_{J}$. Далее мы рассмотрим те новые моменты, которые связаны с появлением у функций зависимости от переменных $e \in S^{n-1}$.

ОПРЕДЕЛЕНИЕ 3.1. Пусть $\gamma(r, e)$ - непрерывная по $r$ функция со значениями в $\mathscr{S}^{\prime}\left(S^{n-1}\right)$. Условимся писать

$$
\gamma(r, e) \sim \rho(r) \Phi(e), \quad r \rightarrow+\infty, \quad \text { на } \mathscr{S}\left(S^{n-1}\right),
$$

где $\Phi(e) \in \mathscr{S}^{\prime}\left(S^{n-1}\right)$, а $\rho(r)$ - положительная функция, если в $\mathscr{S}^{\prime}\left(S^{n-1}\right)$

$$
\frac{1}{\rho(r)} \gamma(r, e) \underset{r \rightarrow+\infty}{\longrightarrow} \Phi(e) .
$$

Лемма 3.2. Пусть $J$ - допустимое множество вещественных чисел $u$ $\rho(k)$ - автомодельная функиия порядка $\beta$, причем $-\beta-1 \notin J$. Пусть задань числа $\ell \in \mathbb{R}, N \in \mathbb{Z}_{+}$, функция $\gamma(r, e)$ со значениями в $\mathscr{S}^{\prime}\left(S^{n-1}\right)$, непрерывная nо $r$ при $r>0$, и обобщенная функиия $\Phi(e) \in \mathscr{S}^{\prime}\left(S^{n-1}\right)$ такие, что на $\mathscr{S}\left(S^{n-1}\right)$

$$
\gamma(r, e) \sim \rho(r) r^{N+\ell} \Phi(e), \quad r \rightarrow+\infty .
$$

Определим обобщенную функиию $F_{1}(r, e) \in W_{J}^{\prime}$ формулой

$$
\left(F_{1}(r, e), \psi(r)\right)=\int_{1}^{\infty}\left(\gamma(r, e),\left(\frac{d}{d r}\right)^{N}\left(r^{-\ell}\left(\psi(r, e)-\sum_{\substack{\lambda \in J \\ \lambda<-\beta-1}} C_{\lambda}(e) r^{\lambda}\right)\right)\right)_{e} d r
$$

для всех $\psi(r, e) \in W_{J}$. Тогда $F_{1}(r, e) \in A O_{\rho}\left(W_{J}\right)$, причем

$$
\frac{1}{\rho(k)} F_{1}(k r, e) \underset{k \rightarrow \infty}{\longrightarrow}(-1)^{N} N !\left(\begin{array}{c}
\beta+\ell+N \\
N
\end{array}\right) r_{J}^{\beta} \otimes \Phi(e) .
$$

ЗАмЕчАниЕ 1. Утверждение леммы останется справедливым и в том случае, когда $-\beta-1 \in J$ и $-\beta-1-\ell \in \mathbb{Z}_{+}$, а $N>-\beta-1-\ell$. При этом вместо (3.7) имеет место соотношение

$$
\frac{1}{\rho(k)} F_{1}(k r, e) \underset{k \rightarrow \infty}{\longrightarrow}(-1)^{\beta+\ell+N+1}(\beta+\ell+N) !(-\beta-\ell-1) ! \Delta_{-\beta-1}(r) \otimes \Phi(e) .
$$

ЗАмЕчАНИЕ 2. Пусть в условиях леммы $3.2-\beta-1 \in J$ и для некоторой $\varphi_{0}(r, e) \in W_{J}$ справедливо соотношение

$$
\left(\gamma(r, e), C_{-\beta-1}^{\varphi_{0}}(e)\right)_{e} \equiv 0,
$$

где $C_{-\beta-1}^{\varphi_{0}}(e)-$ коэффициент разложения функции $\varphi_{0}(r, e)$ в нуле $($ см. $(1.7))$. Тогда существует

$$
\lim _{k \rightarrow \infty} \frac{1}{\rho(k)}\left(F_{1}(k r, e), \varphi_{0}(r, e)\right) \text {. }
$$


Доказательство леммы 3.2 аналогично доказательству леммы 2.2, а для доказательства замечания 1 следует воспользоваться соотношением, аналогичным соотношению (2.15).

Доказательство замечания 2 проводится аналогично доказательству замечания к лемме 2.2, однако вместо соотношения (2.15) следует воспользоваться тождеством (3.9).

Для пространства $W_{J}$ утверждение 2.1 (случай 1)) принимает следующий вид

УТВЕРЖДЕНИЕ 3.1. Пусть $J$ и $J_{1}$ - допустимые множества вещественнъх чисел, $J \subset J_{1}, u \rho(k)$ - автомодельная функиия порядка $\beta$. Пусть $F(r, e) \in$ $W_{J}^{\prime}, F(r, e) \in A O_{\rho}\left(W_{J}\right)$. Тогда если $-\beta-1 \notin J_{1}$, то $F(r, e)$ продолжается на $W_{J_{1}}$ до $\widehat{F}(r, e) \in A O_{\rho}\left(W_{J_{1}}\right)$ maк, ито на $W_{J_{1}}$

$$
\frac{1}{\rho(k)} \widehat{F}(k r, e) \underset{k \rightarrow \infty}{\longrightarrow} r_{J_{1}}^{\beta} \otimes \Phi(e)
$$

с некоторой $\Phi(e) \in \mathscr{S}^{\prime}\left(S^{n-1}\right)$. Любое другое такое продолжение отличается от $\widehat{F}(r, e)$ на функиию

$$
\sum_{\substack{\lambda \in J_{1} \backslash J \\ \lambda>-\beta-1}} \Delta_{\lambda}(r) \otimes \Phi_{\lambda}(e)
$$

с некоторыми $\Phi_{\lambda}(e) \in \mathscr{S}^{\prime}\left(S^{n-1}\right)$.

ЗАМЕЧАНИЕ. Пусть выполнены условия утверждения 3.1, и для некоторого подпространства $\mathscr{E} \subset \mathscr{S}\left(S^{n-1}\right)$ выполнено условие ортогональности, т.е. в $\mathscr{S}_{J}^{\prime}$

$$
(F(r, e), \varphi(e)) \equiv 0 \quad \forall \varphi(e) \in \mathscr{E} .
$$

Тогда существует ее продолжение $\widehat{F}(r, e) \in A O_{\rho}\left(W_{J_{1}}\right)$, для которого также выполнено условие ортогональности, т.е. для любой $\varphi(e) \in \mathscr{E}$ в $\mathscr{S}_{J_{1}}^{\prime}$ имеем

$$
(\widehat{F}(r, e), \varphi(e)) \equiv 0 .
$$

ДокАЗАТЕЛьство. Прежде всего представим $F$ в следующем виде: $F=$ $F_{1}+F_{0}$, где $\operatorname{supp} F_{1}$ отделен от нуля, а $F_{0}$ имеет компактный носитель и сколь угодно много моментов, равных нулю. При этом можно полагать, что соблюдено условие ортогональности и для $F_{1}$, и для $F_{0}$ (см. лемму 3.1 ). Далее рассуждаем, как и при доказательстве леммы 2.3. Заменяя в этих рассуждениях пространства $\mathscr{S}_{J}, \mathscr{S}_{\varnothing}$ на пространства $W_{J}, W_{J_{\varnothing}}$ соответственно, учитывая условия ортогональности при соответствующем продолжении, из формул, аналогичных (2.15)-(2.17), где $C_{\lambda}$ теперь не числа, а обобщенные функции $C_{\lambda}(e) \in \mathscr{S}\left(S^{n-1}\right)$, получим для $F_{1}$ формулу (3.6) с некоторыми $\gamma(r, e), N$ и $\ell$. Так как $\gamma(r, e)=\left(r^{\ell} F_{1}(r, e)\right)^{(-N)}$, где первообразная берется только по $r$, то условие ортогональности будет выполнено и для $\gamma(r, e)$.

Теперь полагаем $\widehat{F}=\widehat{F}_{1}+\widehat{F}_{0}$, где

$$
\left(\widehat{F}_{1}(r, e), \psi(r, e)\right)=\int_{1}^{\infty}\left(\gamma(r, e),\left(\frac{d}{d r}\right)^{(-N)}\left[r^{-\ell}\left(\psi(r, e)-\sum_{\substack{\lambda \in J_{1} \\ \lambda<-\beta-1}} C_{\lambda}(e) r^{\lambda}\right)\right]\right)_{e} d r
$$


с той же $\gamma(r, e)$, а $\widetilde{F}_{0}(r, e)$ - продолжение $F_{0}(r, e)$ с $W_{J}$ на $W_{J_{1}}$ такое, что

$$
\left(\widetilde{F}_{0}(r, e), \varphi(e)\right)_{e}=0, \quad \varphi \in \mathscr{E},
$$

как элемент $\mathscr{S}_{J_{1}}^{\prime}$ и для $\lambda \in J_{1}, \lambda<-\beta-1$,

$$
\left(\widehat{F}_{0}(r, e), r^{\lambda}\right)_{r} \equiv 0
$$

Покажем, что такое продолжение возможно. Докажем сначала, что существует продолжение $\widehat{\widehat{F}}_{0}$, удовлетворяющее условию (3.10). Действительно, пусть $\widetilde{F}_{0}(r, e)$ какое-то продолжение $F_{0}$ на $W_{J_{1}}$. Тогда для любой $\varphi \in \mathscr{E}$ имеем $\left(\widetilde{F}_{0}(r, e), \varphi(e)\right)_{e}=0$ как элемент $\mathscr{S}_{J}^{\prime}$ и поэтому

$$
\left(\widetilde{F}_{0}(r, e), \varphi(e)\right)_{e}=\sum_{\substack{\lambda \in J_{1} \backslash J \\ \lambda \leqslant M}} A_{\lambda}(\varphi) \triangle_{\lambda}(r), \quad \varphi \in \mathscr{E}
$$

как элемент $\mathscr{S}_{J_{1}}$. Из конечности порядка функционала $\widetilde{F}_{0}$ следует, что $M$ не зависит от $\varphi \in \mathscr{E}$, а $A_{\lambda}(\varphi)$ - непрерывные функционалы на $\mathscr{E}$. Продолжим их до обобщенных функций $A_{\lambda}(e)$ на все $\mathscr{S}\left(S^{n-1}\right)$ и положим

$$
\widehat{\widehat{F}}_{0}(r, e)=\widetilde{F}_{0}(r, e)-\sum_{\substack{\lambda \in J_{1} \backslash J \\ \lambda \leqslant M}} A_{\lambda}(e) \triangle_{\lambda}(r) .
$$

Нетрудно видеть, что $\widehat{\widehat{F}}_{0}$ удовлетворяет условию (3.14). Далее положим

$$
\widehat{F}_{0}(r, e)=\widehat{\widehat{F}}_{0}(r, e)-\sum_{\substack{\lambda \in J_{1} \backslash J \\ \lambda<-\beta-1}} M_{\lambda}[\widehat{\widehat{F}}](e) \triangle_{\lambda}(r) .
$$

Легко проверяется, что $\widehat{F}_{0}$ удовлетворяет нужным соотношениям.

Из леммы 3.2 следует, что $\widehat{F} \in A O_{\rho}\left(W_{J_{1}}\right)$ и для $\widehat{F}$ выполнено условие ортогональности. Утверждение 3.1 доказано.

Так же как и в случае пространства $\mathscr{S}_{J}($ см. (2.30)), доказывается, что любая однородная по $r$ степени $\beta$ обобщенная функция $F(r, e) \in W_{J}^{\prime}$ имеет вид

$$
F(r, e)= \begin{cases}r_{J}^{\beta} \otimes \Phi(e), & \text { если }-\beta-1 \notin J, \\ \triangle_{-\beta-1}(r) \otimes \Phi(e), & \text { если }-\beta-1 \in J,\end{cases}
$$

с некоторой $\Phi(e) \in \mathscr{S}^{\prime}\left(S^{n-1}\right)$.

Теорема 2.1 для пространства $W_{J}$ примет следующий вид.

Теорема 3.1. Пусть $F(r, e) \in W_{J}^{\prime}, \rho(k)$ - автомодельная функиия порядка $\beta, J$ - допустимое множество. Пусть число $\nu$ таково, что

$$
\begin{cases}-\beta-1-\nu \notin \mathbb{Z}_{+}, & \text {если }-\beta-1 \notin J \\ -\beta-1-\nu \in \mathbb{Z}_{+}, & \text {если }-\beta-1 \in J\end{cases}
$$


Тогда для того чтобъ $F(r, e) \in A O_{\rho}\left(W_{J}\right)$, необходимо и достаточно, чтоби существовали $N(N>-\beta-1$ в случае $-\beta-1 \in J)$, функция $\gamma(r, e)$ со значениями в $\mathscr{S}^{\prime}\left(S^{n-1}\right)$, непрерывная по $r$ при $r>0$, и обобщенная функиия $\Phi(e) \in \mathscr{S}^{\prime}\left(S^{n-1}\right)$, для которых на $\mathscr{S}\left(S^{n-1}\right)$

$$
\gamma(r, e) \sim r^{\nu+N} \rho(r) \Phi(e), \quad r \rightarrow+\infty
$$

такие, что для любой основной функции $\psi(r, e) \in W_{J}$

$$
\begin{aligned}
(F(r, e), \psi(r, e))= & \int_{1}^{\infty}\left(\gamma(r, e),\left(\frac{d}{d r}\right)^{N}\left[r^{-\nu}\left(\psi(r, e)-\sum_{\substack{\lambda \in J \\
\lambda<-\beta-1}} C_{\lambda}(e) r^{\lambda}\right)\right]\right)_{e} d r \\
& +\left(F_{0}(r, e), \psi(r, e)\right)
\end{aligned}
$$

где $F_{0}(r, e) \in W_{J}^{\prime}$, имеет компактный носитель $($ по $r)$ и $M_{\lambda}\left[F_{0}\right](e)=0$ для $\lambda \in J, \lambda \leqslant-\beta-1$.

ЗАмечАниЕ. Пусть $\mathscr{E}-$ некоторое подпространство $\mathscr{S}\left(S^{n-1}\right)$. Если в условиях теоремы 3.1 полагать, что для $\varphi(e) \in \mathscr{E}$

$$
(F(r, e), \varphi(e))=0
$$

то можно считать, что $(\gamma(r, e), \varphi(e))=0$.

Далее для описания обобщенных функций из $A O_{\rho}\left(V_{J, \mathscr{F}}\right)$, где пространство $V_{J, \mathscr{F}}$ определяется формулой (1.9), нам понадобятся две леммы.

Лемма 3.3. Пусть $J$ - допустимое множество, $F(r, e) \in W_{J}^{\prime}, \rho(k)$ - автомодельная функиия порядка $\beta$, где $-\beta-1 \notin J$. Тогда $F(r, e) \in A O_{\rho}\left(V_{J, \mathscr{F}}\right)$ только в том случае, когда существует $\widehat{F}(r, e) \in A O_{\rho}\left(W_{J}\right)$ такая, что на $V_{J, \mathscr{F}}$

$$
\widehat{F}(r, e)=F(r, e) .
$$

ДокАЗАТЕЛЬство. Сначала напомним, что если $J_{\varnothing}=\varnothing$, то все функции из $W_{J_{\varnothing}}$ имеют в нуле нуль (по $r$ ) бесконечного порядка, так что $W_{J_{\varnothing}} \subset W_{J}$. Пусть $F(r, e) \in A O_{\rho}\left(V_{J, \mathscr{F}}\right)$. Тогда $F(r, e) \in A O_{\rho}\left(W_{J_{\varnothing}}\right)$, так как $W_{J_{\varnothing}} \subset V_{J, \mathscr{F}}$. Следовательно, по утверждению $3.1 F(r, e)$ продолжается на $W_{J}$ до $G(r, e) \in$ $A O_{\rho}\left(W_{J}\right)$. Так как $F$ и $G$ совпадают на $W_{J_{\varnothing}}$, то они отличаются друг от друга на обобщенную функцию, сосредоточенную в нуле, т.е. для некоторого $M$

$$
G(r, e)-F(r, e)=\sum_{\substack{\lambda \in J \\ \lambda<M}} \Delta_{\lambda}(r) \otimes \Phi_{\lambda}(e)=\left(\sum_{\substack{\lambda \in J \\ \lambda<-\beta-1}}+\sum_{\substack{\lambda \in J \\-\beta-1<\lambda<M}}\right) \Delta_{\lambda}(r) \otimes \Phi_{\lambda}(e) .
$$

Второе слагаемое справа обладает тривиальной квазиасимптотикой относительно $\rho(k)$. Положим

$$
\widehat{F}(r, e)=G(r, e)-\sum_{\substack{\lambda \in J \\-\beta-1<\lambda<M}} \Delta_{\lambda}(r) \otimes \Phi_{\lambda}(e) .
$$


Тогда

$$
\widehat{F}(r, e)-F(r, e)=\sum_{\substack{\lambda \in J \\ \lambda<-\beta-1}} \Delta_{\lambda}(r) \otimes \Phi_{\lambda}(e) .
$$

Покажем, что $\widehat{F}(r, e)=F(r, e)$ на $V_{J, \mathscr{F}}$. Так как $\widehat{F} \in A O_{\rho}\left(W_{J}\right)$, а $F \in A O_{\rho}\left(V_{J, \mathscr{F}}\right)$, то $\widehat{F}-F \in A O_{\rho}\left(V_{J, \mathscr{F}}\right)$. Для $\psi(r, e)=r^{\lambda} \eta(r) \chi(e) \in V_{J, \mathscr{F}}$, где $\lambda \in J, \lambda<-\beta-1$, a $\chi(e)$ - произвольная функция из $\mathscr{E}_{\lambda}$, имеем

$$
\begin{aligned}
\frac{1}{\rho(k)} & (\widehat{F}(k r, e)-F(k r, e), \psi(r, e))=\frac{1}{\rho(k)}\left(\Phi_{\lambda}(e) \triangle_{\lambda}(k r), r^{\lambda} \eta(r) \chi(e)\right) \\
& =\frac{1}{k^{\lambda+1} \rho(k)}\left(\Phi_{\lambda}(e), \chi(e)\right) \underset{k \rightarrow \infty}{\longrightarrow} \text { const. }
\end{aligned}
$$

Так как $\lambda<-\beta-1$, то $k^{\lambda+1} \rho(k) \rightarrow 0$ при $k \rightarrow \infty$ и, следовательно, $\left(\Phi_{\lambda}(e), \chi(e)\right)=0$. Так как $\chi(e)-$ произвольная функция из $\mathscr{E}_{\lambda}$, то все коэффициенты $\Phi_{\lambda}(e)$ при $\lambda<-\beta-1$ ортогональны соответствующим $\mathscr{E}_{\lambda}$. Это и означает, что $(\widehat{F}-F, \psi)=0$, если $\psi \in V_{J, \mathscr{F}}$.

Обратное утверждение тривиально. Лемма доказана.

Лемма 3.4. Пусть $\rho(k)$ - автомодельная функиия порядка $\beta,-\beta-1 \in J, u$

$$
F(r, e) \in W_{J}^{\prime}, \quad F(r, e) \in A O_{\rho}\left(V_{J, \mathscr{F}}\right) .
$$

Тогда существует $h(r, e) \in W_{J}^{\prime}$, имеющая следующие свойства:

1) для любой $\varphi(e) \in \mathscr{E}_{-\beta-1}$ справедливо тождество

$$
(F(r, e)-h(r, e), \varphi(e)) \equiv 0
$$

2) существуют $\Phi_{\lambda}(e) \in \mathscr{S}^{\prime}\left(S^{n-1}\right), \lambda \in J, \lambda<-\beta-1$, такие, ито

$$
\widehat{h}(r, e) \equiv h(r, e)-\sum_{\substack{\lambda \in J \\ \lambda<-\beta-1}} \Phi_{\lambda}(e) \Delta_{\lambda}(r) \in A O_{\rho}\left(W_{J}\right) .
$$

ДокАзАтЕЛЬСтво. Так как $F \in A O_{\rho}\left(V_{J, \mathscr{F}}\right)$, т.е.

$$
\frac{1}{\rho(k)} F(k r, e) \underset{k \rightarrow \infty}{\longrightarrow} G(r, e)
$$

на $V_{J, \mathscr{F}}$, а $V_{J, \mathscr{F}}$ замкнуто в $W_{J}$, то эта сходимость имеет место в некотором $V_{J, \mathscr{F}}^{M}\left(\right.$ пополнение $V_{J, \mathscr{F}}$ по норме $\mathscr{P}_{M}$, см. (1.18)). Учитывая конечность порядка $F(r, e)$ на $W_{J}$ и увеличивая $M$, если нужно, можно также считать, что $F(r, e) \in\left(W_{J}^{M}\right)^{\prime}$. Так как $\mathscr{E}_{-\beta-1} \subset \mathscr{S}\left(S^{n-1}\right)$ - ядерное пространство, то существуют число $N$, ограниченное семейство $\left\{\varphi_{m}(e) \in \mathscr{E}_{-\beta-1}^{M}, m=1,2, \ldots\right\}$, ограниченная последовательность функционалов $\left\{f_{m} \in\left(\mathscr{E}_{-\beta-1}\right)^{\prime}\right\}$ и семейство чисел $\left\{\lambda_{m}\right\}, \sum_{m=1}^{\infty}\left|\lambda_{m}\right|<\infty$, таких, что

$$
\psi(e)=\sum_{m=1}^{\infty} \lambda_{m}\left(f_{m}(e), \psi(e)\right) \varphi_{m}(e) \quad \forall \psi(e) \in \mathscr{E}_{-\beta-1}^{N} .
$$


По теореме Хана-Банаха семейство функционалов $\left\{f_{m} \in\left(\mathscr{E}_{-\beta-1}^{N}\right)^{\prime}\right\}$ может быть продолжено до ограниченного семейства функционалов на $\mathscr{S}^{N}\left(S^{n-1}\right)$, в частности, до ограниченного семейства на $\mathscr{S}\left(S^{n-1}\right)$.

Теперь для любой функции $\psi(r, e) \in W_{J}$ положим

$$
\begin{gathered}
(h(r, e), \psi(r, e))=\sum_{m=1}^{\infty} \lambda_{m}\left(F(r, e), \varphi_{m}(e)\left(f_{m}(e), \psi(r, e)\right)_{e}\right) \\
=\sum_{m=1}^{\infty} \lambda_{m}\left(\left(F(r, e), \varphi_{m}(e)\right)_{e},\left(f_{m}(e), \psi(r, e)\right)_{e}\right)_{r} .
\end{gathered}
$$

Отметим, что $h(r, e)$ корректно определена на всем $W_{J}$, т.е. (3.21) имеет смысл для любой $\psi(r, e) \in W_{J}$. Проверим, что

$$
(F(r, e)-h(r, e), \varphi(e)) \equiv 0 \quad \forall \varphi \in \mathscr{E}_{-\beta-1} .
$$

Действительно, для $\varphi(e) \in \mathscr{E}_{-\beta-1}$ и $\psi(r) \in \mathscr{S}_{J}$ имеем

$$
\begin{aligned}
& (h(r, e), \varphi(e) \psi(r))=\sum_{m=1}^{\infty} \lambda_{m}\left(\left(F(r, e), \varphi_{m}(e)\right)_{e},\left(f_{m}(e), \psi(r) \varphi(e)\right)\right)_{r} \\
& \quad=\left(F(r, e), \sum_{m=1}^{\infty} \lambda_{m}\left(f_{m}(e), \varphi(e)\right) \varphi_{m}(e) \cdot \psi(r)\right)=(F(r, e), \varphi(e) \psi(r)) .
\end{aligned}
$$

Кроме того, $h(r, e) \in A O_{\rho}\left(W_{J_{\varnothing}}\right)$, так как

$$
\frac{1}{\rho(k)}(h(k r, e), \psi(r, e))=\sum_{m=1}^{\infty} \lambda_{m} \frac{1}{\rho(k)}\left(F(k r, e), \varphi_{m}(e)\left(f_{m}(e), \psi(r, e)\right)_{e}\right),
$$

а семейство $\left\{\varphi_{m}(e)\left(f_{m}(e), \psi(r, e)\right)_{e}\right\}$ есть ограниченное семейство в $V_{J, \mathscr{F}}^{M}$, если $\psi(r, e) \in W_{J_{\varnothing}}$.

Заметим также, что $h(r, e)$ обладает квазиасимптотикой относительно $\rho(k)$ на функциях вида $r^{-\beta-1} \eta(r) \varphi(e)$, где $\eta(r) \in \mathscr{S}_{+}$имеет компактный носитель и $\eta(r)=1$ в окрестности нуля, а $\varphi(e) \in \mathscr{S}\left(S^{n-1}\right)$. Действительно, это следует из соотношения

$$
\begin{aligned}
\frac{1}{\rho(k)} & \left(h(k r, e), r^{-\beta-1} \eta(r) \varphi(e)\right) \\
& =\sum_{m=1}^{\infty} \lambda_{m}\left(\frac{1}{\rho(k)}\left(F(k r, e), \varphi_{m}(e)\right)_{e}, r^{-\beta-1} \eta(r)\left(f_{m}(e), \varphi(e)\right)_{e}\right)_{r} \\
& =\sum_{m=1}^{\infty} \lambda_{m}\left(f_{m}(e), \varphi(e)\right) \frac{1}{\rho(k)}\left(F(k r, e), r^{-\beta-1} \eta(r) \varphi_{m}(e)\right)
\end{aligned}
$$

в силу того, что последовательность $\left\{\left(f_{m}(e), \varphi(e)\right)\right\}$ ограничена, a $\left\{r^{-\beta-1} \eta(r) \times\right.$ $\left.\varphi_{m}(e)\right\}$ - ограниченное семейство в $V_{J, \mathscr{F}}^{M}$.

Докажем теперь соотношение (3.19). Положим $J_{1}=J \backslash\{-\beta-1\}$. Так как обобщенная функция $h(r, e) \in A O_{\rho}\left(W_{J_{\varnothing}}\right)$, то по утверждению 3.1 она продолжается до обобщенной функции $G(r, e) \in W_{J_{1}}^{\prime}$, причем $G \in A O_{\rho}\left(W_{J_{1}}\right)$. Поскольку $h$ и $G$ совпадают на $W_{J_{\varnothing}}$, то они отличаются на обобщенную функцию, 
сосредоточенную в нуле, т.е.

$$
G(r, e)-h(r, e)=\sum_{\substack{\lambda \in J \\ \lambda<-\beta-1}} \Phi_{\lambda}(e) \Delta_{\lambda}(r)
$$

с некоторыми $\Phi_{\lambda} \in \mathscr{S}^{\prime}\left(S^{n-1}\right)$ (слагаемые с $\lambda>-\beta-1$ не влияют на квазиасимптотику относительно $\rho(k)$ порядка $\beta$ и мы отнесли их к $G)$. Продолжим $G$ на $W_{J}$, обозначив это продолжение через $\widehat{h}(r, e)$. Для этого достаточно определить его на функциях вида $r^{-\beta-1} \eta(r) \varphi(e)$, где $\eta(r)$ удовлетворяет (1.5), а $\varphi(e) \in \mathscr{S}\left(S^{n-1}\right)$. Положим

$$
\left(\widehat{h}(r, e), r^{-\beta-1} \eta(r) \varphi(e)\right)=\left(h(r, e), r^{-\beta-1} \eta(r) \varphi(e)\right) .
$$

Таким образом, $\widehat{h} \in W_{J}$. Кроме того, $\widehat{h}(r, e) \in A O_{\rho}\left(W_{J}\right)$, так как $\widehat{h}(r, e) \in$ $A O_{\rho}\left(W_{J_{1}}\right)$ и обладает квазиасимптотикой на функциях вида $r^{-\beta-1} \eta(r) \varphi(e)$, где $\varphi(e) \in \mathscr{S}\left(S^{n-1}\right)$, так как $h$ обладает ею. Лемма доказана.

Следующие две теоремы дают описание обобщенных функций из $A O_{\rho}\left(V_{J, \mathscr{F}}\right)$.

Теорема 3.2. Пусть $J$ - допустимое множество, $\rho(k)$ - автомодельная бункция порядка $\beta,-\beta-1 \notin J, F(r, e) \in W_{J}^{\prime}, \nu$ - некоторое число, длякоторого $-\beta-1-\nu \notin \mathbb{Z}_{+}$. Для того чтобъ $F(r, e) \in A O_{\rho}\left(V_{J, \mathscr{F}}\right)$, необходимо и достаточно, чтобы существовали число $N$, функиия $\gamma(r, e)$, непрерывная nо $r$ при $r>0$ со значениями в $\mathscr{S}^{\prime}\left(S^{n-1}\right)$, для которых на $\mathscr{S}\left(S^{n-1}\right)$ с некоторой $\Phi(e) \in \mathscr{S}^{\prime}\left(S^{n-1}\right)$

$$
\gamma(r, e) \sim r^{\nu+N} \rho(r) \Phi(e), \quad r \rightarrow \infty
$$

maкue, чmo

$$
\begin{gathered}
(F(r, e), \psi(r, e))=\int_{1}^{\infty}\left(\gamma(r, e),\left(\frac{d}{d r}\right)^{N}\left[r^{-\nu}\left(\psi(r, e)-\sum_{\substack{\lambda \in J \\
\lambda<-\beta-1}} C_{\lambda}(e) r^{\lambda}\right)\right]\right)_{e} d r \\
+\left(F_{0}(r, e), \psi(r, e)\right) \quad \forall \psi(r, e) \in V_{J, \mathscr{F}}
\end{gathered}
$$

где $F_{0}(r, e) \in W_{J}^{\prime}$, имеет компактный носитель по $r$ и $M_{\lambda}\left[F_{0}\right](e) \equiv 0$ для $\lambda \in J$, $\lambda<-\beta-1$.

Доказательство теоремы непосредственно следует из теоремы 3.1 и леммы 3.1 .

ЗАмЕЧАНИЕ. Если $(F(r, e), \varphi(e)) \equiv 0$ для всех $\varphi(e)$ из некоторого подпространства $\mathscr{S}\left(S^{n-1}\right)$, то можно считать, что $(\gamma(r, e), \varphi(e)) \equiv 0$ и $\left(F_{0}(r, e), \varphi(e)\right) \equiv 0$ для $\varphi(e)$ из того же подпространства (см. лемму 3.1 и замечание к утверждению 3.1$)$.

Теорема 3.3. Пусть $J$ - допустимое множество, $\rho(k)$ - автомодельная функиия порядка $\beta,-\beta-1 \in J, F(r, e) \in W_{J}^{\prime} u \nu$ - некоторое число, для 
которого $-\beta-1-\nu \notin \mathbb{Z}_{+}$. Для того чтобъ $F(r, e) \in A O_{\rho}\left(V_{J, \mathscr{F}}\right)$, необходимо и достаточно, чтобъ

$$
F(r, e)=F_{1}(r, e)+F_{2}(r, e), \quad F_{1}(r, e) \in A O_{\rho}\left(W_{J}\right), \quad F_{2}(r, e) \in W_{J}^{\prime} .
$$

При этом существуют число $N$, функция $\gamma(r, e)$, непрерывная по $r$ при $r>0$ со значениями в $\mathscr{S}^{\prime}\left(S^{n-1}\right)$, такие, что для всех $\varphi(e) \in \mathscr{E}_{-\beta-1}$

$$
\begin{gathered}
(\gamma(r, e), \varphi(e)) \equiv 0, \\
\gamma(r, e) \sim r^{\nu+N} \rho(r) \Phi(e), \quad r \rightarrow \infty, \quad \text { на } \mathscr{S}\left(S^{n-1}\right)
\end{gathered}
$$

с некоторой $\Phi(e) \in \mathscr{S}^{\prime}\left(S^{n-1}\right)$, причем для любой $\psi(r, e) \in V_{J, \mathscr{F}}$

$$
\left(F_{2}(r, e), \psi(r, e)\right)=\int_{1}^{\infty}\left(\gamma(r, e),\left(\frac{d}{d r}\right)^{N}\left[r^{-\nu}\left(\psi(r, e)-\sum_{\substack{\lambda \in J \\ \lambda<-\beta-1}} C_{\lambda}(e) r^{\lambda}\right)\right]\right)_{e} d r
$$

ДоказАтельство. Пусть $F(r, e) \in A O_{\rho}\left(V_{J, \mathscr{F}}\right)$. Согласно лемме 3.2 существует $h(r, e) \in W_{J}^{\prime}$, удовлетворяющая условиям (3.18) и (3.19). Имеем

$$
F(r, e)=F(r, e)-h(r, e)+\Delta(r, e)+\widehat{h}(r, e), \quad \Delta=\sum_{\substack{\lambda \in J \\ \lambda<-\beta-1}} \Phi_{\lambda}(e) \otimes \Delta_{\lambda}(r) .
$$

Так как $\widehat{h} \in A O_{\rho}\left(W_{J}\right)$, то $F-h+\Delta \in A O_{\rho}\left(V_{J, \mathscr{F}}\right)$. Учитывая, что носитель $\triangle$ сосредоточен в нуле, получаем, что

$$
G(r, e) \equiv F(r, e)-h(r, e) \in A O_{\rho}\left(W_{J_{\varnothing}}\right) .
$$

При этом согласно (3.18) имеем $(G(r, e), \varphi(e)) \equiv 0$ для любой $\varphi(e) \in \mathscr{E}_{-\beta-1}$. Положим $J_{1}=J \backslash\{-\beta-1\}$. По утверждению 3.1 обобщенная функция $G(r, e)$ продолжается на $W_{J_{1}}$ до обобщенной функции $\widetilde{G}(r, e) \in A O_{\rho}\left(W_{J_{1}}\right)$, причем $(\widetilde{G}(r, e), \varphi(e)) \equiv 0$ для любой $\varphi \in \mathscr{E}_{-\beta-1}($ см. замечание к утверждению 3.1$)$.

По теореме 3.2 для $\widetilde{G}(r, e)$ справедливо представление

$$
\begin{gathered}
(\widetilde{G}(r, e), \psi(r, e))=\int_{1}^{\infty}\left(\gamma(r, e),\left(\frac{d}{d r}\right)^{N}\left[r^{-\nu}\left(\psi(r, e)-\sum_{\substack{\lambda \in J_{1} \\
\lambda<-\beta-1}} C_{\lambda}(e) r^{\lambda}\right)\right]\right)_{e} d r \\
+\left(\widetilde{G}_{0}(r, e), \psi(r, e)\right), \quad \psi \in W_{J_{1}},
\end{gathered}
$$

где $N, \nu, \gamma(r, e)$ определяются теоремой 3.2 , а $\widetilde{G}_{0}(r, e)$ имеет компактный носитель, большое количество соответствующих моментов, равных нулю, и $\left(\widetilde{G}_{0}(r, e)\right.$, $\varphi(e)) \equiv 0$ для любой $\varphi \in \mathscr{E}_{-\beta-1}$. При этом согласно замечанию к теореме 3.2 выполнено условие (3.29).

Заметим, что интеграл в (3.33) корректно определен для любой $\psi \in W_{J}$ и определяет обобщенную функцию из $W_{J}^{\prime}$, которая в силу (3.29) также принадлежит $A O_{\rho}\left(V_{J, \mathscr{F}}\right)$. Обозначим эту обобщенную функцию через $\widetilde{F}_{2}(r, e)$. Продолжим $\widetilde{G}_{0}(r, e)$ на все $W_{J}$ с сохранением нужного количества нулевых моментов. 
Теперь заметим, что $\widetilde{F}_{2}+\widetilde{G}_{0}$ и $F-h+\triangle$ совпадают на $W_{J_{\varnothing}}$ и принадлежат $A O_{\rho}\left(V_{J, \mathscr{F}}\right)$. Поэтому они отличаются на обобщенную функцию $\Delta_{1}(r, e)$, сосредоточенную в нуле, причем $\Delta_{1}(r, e) \in A O_{\rho}\left(V_{J, \mathscr{F}}\right)$. Другими словами, существуют число $M$, обобщенные функции $\Psi_{\lambda}(e) \in \mathscr{S}\left(S^{n-1}\right), \lambda \in J, \lambda \leqslant M$, такие, что

$$
F-h+\Delta=\widetilde{F}_{2}+\widetilde{G}_{0}+\Delta_{1}, \quad \Delta_{1}(r, e)=\sum_{\substack{\lambda \in J \\ \lambda<M}} \Psi_{\lambda}(e) \otimes \Delta_{\lambda}(r) .
$$

Те слагаемые в сумме для $\triangle_{1}(r, e)$, которые обладают квазиасимптотикой (может даже тривиальной) относительно $\rho(k)$ на всем $W_{J}$, объединим в сумму, которую обозначим через $\triangle_{2}$. Так как $\Delta_{1} \in A O_{\rho}\left(V_{J, \mathscr{F}}\right)$, то для каждого из остальных слагаемых на $V_{J, \mathscr{F}}$ справедливо соотношение

$$
\Psi_{\lambda}(e) \otimes \triangle_{\lambda}(r) \equiv 0,
$$

т.е. $\left(\Psi_{\lambda}(e), \varphi(e)\right)=0, \varphi \in \mathscr{E}_{\lambda}$. Сумму этих слагаемых обозначим через $\triangle_{3}(r, e)$.

Теперь положим

$$
F_{2}(r, e)=\widetilde{F}_{2}(r, e)+\triangle_{3}(r, e), \quad F_{1}(r, e)=\widehat{h}(r, e)+\widetilde{G}_{0}(r, e)+\triangle_{2}(r, e) .
$$

Нетрудно проверить, что $F_{1}$ и $F_{2}$ обладают нужными свойствами.

Обратное утверждение тривиально. Теорема доказана.

Следующее утверждение описывает однородные обобщенные функции на пространстве $V_{J, \mathscr{F}}$.

УТВЕРЖДЕНИЕ 3.2. Пусть $J$ - допустимое множество, обобщенная функиия $F(r, e) \in W_{J}^{\prime}$ и является однородной степени $\beta$ на $V_{J, \mathscr{F}}$. Тогда справедливь следующие утверждения:

1) если $-\beta-1 \notin J$, то существует $\Phi(e) \in \mathscr{S}^{\prime}\left(S^{n-1}\right)$ такая, что

$$
(F(r, e), \psi(r, e))=\left(r_{J}^{\beta} \otimes \Phi(e), \psi(r, e)\right) \quad \forall \psi \in V_{J, \mathscr{F}} ;
$$

2) если - $\beta-1 \in J$, то существуют обобщенные фучнкиии $\Psi(e)$ и $\Phi(e)$ из $\mathscr{S}^{\prime}\left(S^{n-1}\right)$, причем

$$
(\Phi(e), \varphi(e))=0 \quad \forall \varphi(e) \in \mathscr{E}_{-\beta-1},
$$

такие, что для любой функции $\psi(r, e) \in V_{J, \mathscr{F}}$

$$
\begin{aligned}
& (F(r, e), \psi(r, e))=\left(r_{J}^{\beta} \otimes \Phi(e), \psi(r, e)\right)+\left(\triangle_{-\beta-1}(r) \otimes \Psi(e), \psi(r, e)\right) \\
& \quad=\int_{0}^{\infty} r^{\beta}\left(\Phi(e), \psi(r, e)-\sum_{\substack{\lambda \in J \\
\lambda<-\beta-1}} C_{\lambda}(e) r^{\lambda}\right)_{e} d r+\left(\Psi(e), C_{-\beta-1}(e)\right) .
\end{aligned}
$$

Отметим, что в интеграл в (3.37) сходится в силу условия ортогональности (3.36). 
ДокАзАтельство. Пусть сначала $-\beta-1 \notin J$. Так как однородная функция степени $\beta$ - асимптотически однородна относительно $\rho(k)=k^{\beta}$, то по теореме 3.2 для некоторого $\nu$ и для $\psi(r, e) \in V_{J, \mathscr{F}}$ имеем

$$
\begin{gathered}
(F(r, e), \psi(r, e))=\frac{1}{k^{\beta}}(F(k r, e), \psi(r, e))=\frac{1}{k^{\beta+1}}\left(F(r, e), \psi\left(\frac{r}{k}, e\right)\right) \\
=\frac{1}{k^{\beta+1}} \int_{1}^{\infty}\left(\gamma(r, e),\left(\frac{d}{d r}\right)^{N}\left[r^{-\nu}\left(\psi\left(\frac{r}{k}, e\right)-\sum_{\substack{\lambda \in J \\
\lambda<-\beta-1}} \frac{C_{\lambda}(e)}{k^{\lambda}} r^{\lambda}\right)\right]\right)_{e} d r \\
\quad+\frac{1}{k^{\beta}}\left(F_{0}(k r, e), \psi(r, e)\right) .
\end{gathered}
$$

Так как $F_{0}$ имеет достаточно много моментов, равных нулю, то последнее слагаемое в (3.38) стремится к нулю при $k \rightarrow \infty$, а интеграл в (3.38) (после замены переменных $k r^{\prime}=r$ и опускания штрихов) равен

$$
\begin{gathered}
\frac{1}{k^{\beta+\nu+N}} \int_{1 / k}^{\infty}\left(\gamma(k r, e),\left(\frac{d}{d r}\right)^{N}\left[r^{-\nu}\left(\psi(r, e)-\sum_{\substack{\lambda \in J \\
\lambda<-\beta-1}} C_{\lambda}(e) r^{\lambda}\right)\right]\right)_{e} d r \\
\underset{k \rightarrow \infty}{\longrightarrow} \int_{0}^{\infty} r^{\nu+\beta+N}\left(\Phi(e),\left(\frac{d}{d r}\right)^{N}\left[r^{-\nu}\left(\psi(r, e)-\sum_{\substack{\lambda \in J \\
\lambda<-\beta-1}} C_{\lambda}(e) r^{\lambda}\right)\right]\right)_{e} d r .
\end{gathered}
$$

Здесь мы воспользовались соотношением (3.26). Интегрируя теперь по частям, получаем соотношение (3.35).

Пусть теперь $-\beta-1 \in J$. Пользуясь теоремой 3.3 , для любой функции $\psi(r, e) \in V_{J, \mathscr{F}}$ имеем

$$
F(r, e)=F_{1}(r, e)+F_{2}(r, e), \quad F_{1} \in A O_{\rho}\left(W_{J}\right),
$$

и согласно случаю 3 ) утверждения 2.1 ("тензорно умноженному на $\mathscr{S}\left(S^{n-1}\right)$ "), существует обобщенная функция $\Psi(e) \in \mathscr{S}^{\prime}\left(S^{n-1}\right)$ такая, что

$$
F_{1}(r, e)=\frac{1}{k^{\beta}} F_{1}(k r, e) \underset{k \rightarrow \infty}{\longrightarrow} \triangle_{-\beta-1}(r) \otimes \Psi(e)
$$

на $W_{J}$ и, следовательно, и на $V_{J, \mathscr{F}}$. Для $F_{2}$, пользуясь $(3.31)$ и учитывая $(3.29)$ и $(3.30)$, имеем для любой $\psi(r, e) \in V_{J, \mathscr{F}}$

$$
\begin{aligned}
& \left(F_{2}(r, e), \psi(r, e)\right)=\frac{1}{k^{\beta}}\left(F_{2}(k r, e), \psi(r, e)\right)=\frac{1}{k^{\beta+1}}\left(F_{2}(r, e), \psi\left(\frac{r}{k}, e\right)\right) \\
& =\frac{1}{k^{\beta+1}} \int_{1}^{\infty}\left(\gamma(r, e),\left(\frac{d}{d r}\right)^{N}\left[r^{-\nu}\left(\psi\left(\frac{r}{k}, e\right)-\sum_{\substack{\lambda \in J \\
\lambda<-\beta-1}} \frac{C_{\lambda}(e)}{k^{\lambda}} r^{\lambda}\right)\right]\right)_{e} d r \\
& =\frac{1}{k^{\beta+\nu+N}} \int_{\frac{1}{k}}^{\infty}\left(\gamma(k r, e),\left(\frac{d}{d r}\right)^{N}\left[r^{-\nu}\left(\psi(r, e)-\sum_{\substack{\lambda \in J \\
\lambda<-\beta-1}} C_{\lambda}(e) r^{\lambda}\right)\right]\right)_{e} d r \\
& \underset{k \rightarrow \infty}{\longrightarrow} \int_{0}^{\infty} r^{\nu+\beta+N}\left(\Phi_{1}(e),\left(\frac{d}{d r}\right)^{N}\left[r^{-\nu}\left(\psi(r, e)-\sum_{\substack{\lambda \in J \\
\lambda<-\beta-1}} C_{\lambda}(e) r^{\lambda}\right)\right]\right)_{e} d r .
\end{aligned}
$$


Интегрируя теперь по частям, получим вместе с (3.39) соотношения (3.36) и (3.37). Утверждение доказано.

ЗАмЕчАниЕ. Учитывая условие ортогональности (3.36), можно в (3.37) под знак интеграла (в круглые скобки) добавить слагаемое $-C_{-\beta-1}(e) r^{-\beta-1} \Theta(1-r)$. Теперь утверждение 3.2 можно сформулировать следующим образом:

Для того чтобы обобщенная функиия $F(r, e) \in W_{J}^{\prime}$ была однородной степени $\beta$ на $V_{J, \mathscr{F}}$, необходимо и достаточно, чтобъ на $V_{J, \mathscr{F}}$ она представлялась в таком виде:

$$
F(r, e)= \begin{cases}r_{J}^{\beta} \otimes \Psi(e), & \text { если }-\beta-1 \notin J, \\ \triangle_{-\beta-1}(r) \otimes \Psi(e)+r_{J}^{\beta} \otimes \Phi(e), & \text { если }-\beta-1 \in J,\end{cases}
$$

для некоторых обобщенных функций $\Psi(e)$ и $\Phi(e)$ из $\mathscr{S}^{\prime}\left(S^{n-1}\right)$, при этом $\Phi(e)$ должна быть ортогональна множеству функиий $\mathscr{E}_{-\beta-1}$.

Отметим, что все результаты этого параграфа останутся справедливыми, если пространство $\mathscr{S}\left(S^{n-1}\right)$ заменить на любое ядерное пространство Фреше.

\section{§ 4. Представление асимптотически однородных обобщенных функций по специальным группам преобразований}

В этом параграфе мы опишем асимптотически однородные функции из пространства $\mathscr{S}^{\prime}\left(\mathbb{R}^{n}\right)$ по группе преобразований, определяемой вектором $a=\left(a_{1}\right.$, $\left.\ldots, a_{n}\right) \in \mathbb{R}_{+}^{n}$ (см. определение 0.2). Пространства $W_{J_{a}}$ и $V_{a}$ введены в $\S 1$. Предварительно сделаем следующее

ЗАмЕчАниЕ 4.1. Пусть $f_{0}(t) \in \mathscr{S}^{\prime}\left(\mathbb{R}^{n}\right)$ и имеет компактный носитель. Если $F_{0}(r, e)$ - ее обобщенное сферическое представление и

$$
M_{\lambda}\left[F_{0}\right](e)=0, \quad \lambda \in J_{a}, \quad \lambda \leqslant M,
$$

то

$$
\left(f_{0}(t), t^{j}\right)=0, \quad(a, j) \leqslant M .
$$

Это следует из соотношения

$$
\left(f_{0}(t), t^{j}\right)=\left(F_{0}(r, e), r^{(a, j)} E_{j}(e)\right) .
$$

Верно и обратное. Если выполнено соотношение (4.2), то существует обобщенное сферическое представление $F_{0}(r, e)$ с компактным носителем, для которого выполнено соотношение (4.1).

Действительно, пусть $F_{1}(r, e)$ - какое-либо обобщенное сферическое представление $f_{0}$. Так как

$$
\left(f_{0}(t), t^{j}\right)=\left(F_{1}(r, e), r^{a_{1} j_{1}} e_{1}^{j_{1}} \cdots r^{a_{n}} e_{n}^{j_{n}}\right)=\left(F_{1}(r, e), r^{(a, j)} E_{j}(e)\right),
$$

то $\left(F_{1}(r, e), r^{\lambda} \varphi(e)\right)=0$ при $\lambda \in J_{a}, \lambda \leqslant M, \varphi(e) \in \mathscr{E}_{\lambda}^{a}$, т.е. $M_{\lambda}\left[F_{1}\right](e) \equiv 0$ на $\mathscr{E}_{\lambda} a$. Теперь в качестве искомого представления можно взять

$$
F_{0}(r, e)=F_{1}(r, e)-\sum_{\substack{\lambda \in J_{a} \\ \lambda \leqslant M}} M_{\lambda}\left[F_{1}\right](e) \otimes \Delta_{\lambda}(r) .
$$


Следующие теоремы дают описание обобщенных функций

$$
f(t) \in A O_{\rho}^{a}\left(\mathscr{S}\left(\mathbb{R}^{n}\right)\right) .
$$

ТеОрема 4.1. Пусть $\rho(k)$ - автомодельная функиия порядка $\alpha$, вектор $a=\left(a_{1}, \ldots, a_{n}\right) \in \mathbb{R}_{+}^{n}$, причем

$$
\alpha \notin\left\{-(a, \bar{n}), \bar{n}=\left(n_{1}, \ldots, n_{n}\right) \in \mathbb{N}^{n}\right\} .
$$

Пусть $\nu$ - произвольное число, для которого $-\nu-\alpha-|a| \notin \mathbb{Z}_{+}$. Для того чтобы $f(t) \in A O_{\rho}^{a}\left(\mathscr{S}\left(\mathbb{R}^{n}\right)\right)$, необходимо и достаточно, чтобы

$$
f(t)=f_{1}(t)+f_{0}(t)
$$

где $f_{0}$ и $f_{1}$ удовлетворяют следуюшим условиям: $f_{0}(t) \in \mathscr{S}^{\prime}\left(\mathbb{R}^{n}\right)$, имеет компактный носитель и

$$
\left(f_{0}(t), t^{j}\right)=0 \quad \partial \Omega я(a, j) \leqslant-\alpha-|a|,
$$

существуют $N \in \mathbb{Z}_{+}$, функиия $\gamma(r, e)$, непрерьвная по $r$ при $r>0$ со значениями в $\mathscr{S}^{\prime}\left(S^{n-1}\right)$ и асимптотикой

$$
\gamma(r, e) \sim r^{\nu+N+|a|-1} \rho(r) \Psi(e), \quad r \rightarrow \infty,
$$

для некоторой $\Psi(e) \in \mathscr{S}^{\prime}\left(S^{n-1}\right)$, такие, что

$$
\begin{aligned}
& \left(f_{1}(t), \varphi(t)\right)=\int_{1}^{\infty}\left(\gamma(r, e),\left(\frac{d}{d r}\right)^{N}\left[r ^ { - \nu } \left(\varphi\left(r^{a_{1}} e_{1}, \ldots, r^{a_{n}} e_{n}\right)\right.\right.\right. \\
& \left.\left.\left.\quad-\sum_{\substack{(m, a)<-\alpha-|a| \\
m \in \mathbb{Z}_{+}^{n}}} \frac{1}{m !} r^{(m, a)} \varphi^{(m)}(0) E_{m}(e)\right)\right]\right)_{e} d r \quad \forall \varphi(t) \in \mathscr{S}\left(\mathbb{R}^{n}\right) .
\end{aligned}
$$

ДоказАтельство. Пусть $f(t) \in A O_{\rho}^{a}\left(\mathscr{S}\left(\mathbb{R}^{n}\right)\right)$ и $F(r, e)$ - ее обобщенное сферическое представление. Тогда согласно утверждению 1.2 ее обобщенное сферическое представление $F(r, e) \in A O_{\rho_{1}}\left(V_{a}\right)$, где $\rho_{1}(k)=k^{|a|-1} \rho(k)$ - автомодельная функция порядка $\beta=\alpha+|a|-1$. В силу (4.1) имеем $-\beta-1 \notin J_{a}$ и доказательство теоремы немедленно следует из теоремы 3.2 и замечания 4.1, с учетом того, что для функции $\varphi\left(r^{a_{1}} e_{1}, \ldots, r^{a_{n}} e_{n}\right)$ справедливо соотношение

$$
\sum_{\substack{\lambda<-\beta-1 \\ \lambda \in J_{a}}} C_{\lambda}(e) r^{\lambda}=\sum_{\substack{(m, a)<-\alpha-|a| \\ m \in \mathbb{Z}_{+}^{n}}} \frac{1}{m !} r^{(m, a)} \varphi^{(m)}(0) E_{m}(e) .
$$

Обратное утверждение очевидно. Теорема доказана.

ЗАмЕчАнИЕ. Если $\alpha>-|a|$, то формулировка теоремы 4.1 упрощается и принимает следующий вид.

Для того чтобы $f(t) \in A O_{\rho}\left(\mathscr{S}\left(\mathbb{R}^{n}\right)\right)$, необходимо и достаточно, чтобы существовали число $N \in \mathbb{Z}_{+}$и функция $\gamma(r, e)$, непрерывная по $r$ со значениями в $\mathscr{S}^{\prime}\left(S^{n-1}\right)$ и асимптотикой

$$
\gamma(r, e) \sim r^{N+|a|-1} \rho(r) \Psi(e), \quad r \rightarrow \infty,
$$


для некоторой $\Psi(e) \in \mathscr{S}^{\prime}\left(S^{n-1}\right)$, такие, что для любой $\varphi(t) \in \mathscr{S}\left(\mathbb{R}^{n}\right)$ с носителем $\operatorname{supp} \varphi \subset\{|t|>1\}$ выполнено равенство

$$
(f(t), \varphi(t))=\int_{1}^{\infty}\left(\gamma(r, e),\left(\frac{d}{d r}\right)^{N} \varphi\left(r^{a_{1}} e_{1}, \ldots, r^{a_{n}} e_{n}\right)\right)_{e} d r .
$$

ТеОрема 4.2. Пусть $\rho(k)$ - автомодельная функиия порядка $\alpha$, вектор $a=\left(a_{1}, \ldots, a_{n}\right) \in \mathbb{R}_{+}^{n}$, причем

$$
\alpha \in\left\{-(a, \bar{n}): \bar{n}=\left(n_{1}, \ldots, n_{n}\right) \in \mathbb{N}^{n}\right\},
$$

и произвольные числа $\mu$ и таковы, что

$$
-\mu-\alpha-|a| \notin \mathbb{Z}_{+}, \quad-\nu-\alpha-|a| \in \mathbb{Z}_{+} .
$$

Для того чтобъ $f(t) \in A O_{\rho}^{a}\left(\mathscr{S}\left(\mathbb{R}^{n}\right)\right)$, необходимо и достаточно, чтобъ

$$
f(t)=f_{1}(t)+f_{2}(t)+f_{0}(t)
$$

где обобщенные функиии $f_{i}(t) \in \mathscr{S}^{\prime}\left(\mathbb{R}_{n}\right), i=0,1,2$, удовлетворяют следующим условиям: $f_{0}(t) \in \mathscr{S}^{\prime}\left(\mathbb{R}^{n}\right)$, имеет компактный носитель и

$$
\left(f_{0}(t), t^{j}\right)=0 \quad \text { для }(a, j) \leqslant-\alpha-|a|,
$$

существуют $N \in \mathbb{Z}_{+}(N>-\alpha-|a|-\nu)$, функиия $\gamma(r, e)$, непрерьвная по $r$ при $r>0$ со значениями в $\mathscr{S}^{\prime}\left(S^{n-1}\right)$ и асимптотикой

$$
\gamma(r, e) \sim r^{\nu+N+|a|-1} \rho(r) \Psi(e), \quad r \rightarrow \infty, \quad \text { на } \mathscr{S}\left(S^{n-1}\right)
$$

с некоторой $\Psi(e) \in \mathscr{S}^{\prime}\left(S^{n-1}\right)$, причем

$$
\begin{aligned}
& \left(f_{1}(t), \varphi(t)\right)=\int_{1}^{\infty}\left(\gamma(r, e),\left(\frac{d}{d r}\right)^{N}\left[r ^ { - \nu } \left(\varphi\left(r^{a_{1}} e_{1}, \ldots, r^{a_{n}} e_{n}\right)\right.\right.\right. \\
& \left.\left.\left.-\sum_{\substack{(m, a)<-\alpha-|a| \\
m \in \mathbb{Z}_{+}^{n}}} \frac{1}{m !} r^{(m, a)} \varphi^{(m)}(0) E_{m}(e)\right)\right]\right)_{e} d r \quad \forall \varphi(t) \in \mathscr{S}\left(\mathbb{R}^{n}\right) ;
\end{aligned}
$$

существуют $N \in \mathbb{Z}_{+}$, бункиия $\omega(r, e)$, непрерьвная по $r$ при $r>0$ со значениями в $\mathscr{S}^{\prime}\left(S^{n-1}\right)$ и асимптотикой

$$
\omega(r, e) \sim r^{\mu+N+|a|-1} \rho(r) \Phi(e), \quad r \rightarrow \infty, \quad \text { на } \mathscr{S}\left(S^{n-1}\right)
$$

с некоторой $\Phi(e) \in \mathscr{S}^{\prime}\left(S^{n-1}\right)$,

$$
\left(\omega(r, e), E_{j}(e)\right) \equiv 0 \quad \text { для всех } j \operatorname{maких,~ито~}(j, a)=-\alpha-|a|,
$$

причем

$$
\begin{aligned}
& \left(f_{2}(t), \varphi(t)\right)=\int_{1}^{\infty}\left(\omega(r, e),\left(\frac{d}{d r}\right)^{N}\left[r ^ { - \mu } \left(\varphi\left(r^{a_{1}} e_{1}, \ldots, r^{a_{n}} e_{n}\right)\right.\right.\right. \\
& \left.\left.\left.\quad-\sum_{\substack{(m, a)<-\alpha-|a| \\
m \in \mathbb{Z}_{+}^{n}}} \frac{1}{m !} r^{(m, a)} \varphi^{(m)}(0) E_{m}(e)\right)\right]\right)_{e} d r \quad \forall \varphi(t) \in \mathscr{S}\left(\mathbb{R}^{n}\right) .
\end{aligned}
$$


Доказательство проводится по той же схеме, что и доказательство теоремы 4.1, только теперь вместо теоремы 3.2 следует воспользоваться теоремами 3.3 и 3.1.

Обратное утверждение очевидно. Теорема доказана.

ЗАмечАниЕ 4.2. Нетрудно видеть, что теоремы 4.1 и 4.2 останутся справедливыми, если вместо непрерывности функций $\gamma(r, e)$ и $\omega(r, e)$, как функций со значениями в $\mathscr{S}^{\prime}\left(S^{n-1}\right)$, потребовать лишь их слабую измеримость, т.е. потребовать, чтобы для любой функции $\varphi(e) \in \mathscr{S}\left(S^{n-1}\right)$ функции $(\gamma(r, e), \varphi(e))_{e}$ и $(\omega(r, e), \varphi(e))_{e}$ были измеримыми функциями по $r$.

Далее мы опишем квазиоднородные обобщенные функции из $\mathscr{S}^{\prime}\left(\mathbb{R}^{n}\right)$, точнее, функции, однородные относительно группы преобразований, определяемой вектором $a \in \mathbb{R}_{+}^{n}$. Для такой квазиоднородной функции $f(t)$ степени $\alpha$ имеем

$$
\begin{aligned}
& (f(t), \varphi(t))=\frac{1}{k^{\alpha}}\left(f\left(k^{a_{1}} t_{1}, \ldots, k^{a_{n}} t_{n}\right), \varphi(t)\right) \\
& \quad=\frac{1}{k^{\alpha+|a|-1}}\left(F(k r, e), \varphi\left(r^{a_{1}} e_{1}, \ldots, r^{a_{n}} e_{n}\right)\right) \\
& \quad=\left(F(r, e), \varphi\left(r^{a_{1}} e_{1}, \ldots, r^{a_{n}} e_{n}\right)\right) \quad \forall \varphi \in \mathscr{S}\left(\mathbb{R}^{n}\right)
\end{aligned}
$$

(см. (1.20)). Поэтому ее обобщенное сферическое представление является однородной по $r$ функцией степени $\beta=\alpha+|a|-1$ на $V_{a}$. Из (3.40) для любой $\varphi(t) \in \mathscr{S}\left(\mathbb{R}^{n}\right)$ получаем

$$
(f(t), \varphi(t))=\left\{\begin{array}{r}
\left(r_{J_{a}}^{\alpha+|a|-1} \otimes \Psi(e), \varphi\left(r^{a_{1}} e_{1}, \ldots, r^{a_{n}} e_{n}\right)\right), \quad \text { если }-\alpha-|a| \notin J_{a} \\
\left(\triangle_{-\alpha-|a|}(r) \otimes \Psi(e)+r_{J_{a}}^{\alpha+|a|-1} \otimes \Phi(e), \varphi\left(r^{a_{1}} e_{1}, \ldots, r^{a_{n}} e_{n}\right)\right), \\
\text { если }-\alpha-|a| \in J_{a} .
\end{array}\right.
$$

Здесь $\Psi(e)$ и $\Phi(e)$ - обобщенные функции из $\mathscr{S}^{\prime}\left(S^{n-1}\right)$, причем $(\Phi(e), \psi(e))=0$ при любой $\psi(e) \in \mathscr{E}_{-\alpha-|a|}$.

Таким образом, справедливо следующее

УТВЕРЖДЕНИЕ 4.3. Для того чтобъ обобщенная функиия $f(t) \in \mathscr{S}^{\prime}\left(\mathbb{R}^{n}\right)$ была однородной степени $\alpha$ относительно группь преобразований, определяемой вектором $a \in \mathbb{R}_{+}^{n}$, необходимо и достаточно, чтобы для нее выполнялись следующие условия:

1) если $-\alpha \notin\left\{(a, \ell), \ell \in \mathbb{N}^{n}\right\}$, то существует бункиия $\Phi(e) \in \mathscr{S}^{\prime}\left(S^{n-1}\right)$ такая, что

$$
\begin{aligned}
& (f(t), \varphi(t))=\int_{0}^{\infty} r^{\alpha+|a|-1}\left(\Phi(e),\left(\varphi\left(r^{a_{1}} e_{1}, \ldots, r^{a_{n}} e_{n}\right)\right.\right. \\
& \left.\left.-\sum_{\substack{(m, a)<-\alpha-|a| \\
m \in \mathbb{Z}_{+}^{n}}} r^{(m, a)} \frac{1}{m !} \varphi^{(m)}(0) E_{m}(e)\right)\right)_{e} d r \quad \forall \varphi(t) \in \mathscr{S}\left(\mathbb{R}^{n}\right) ;
\end{aligned}
$$


2) если $-\alpha \in\left\{(a, \ell), \ell \in \mathbb{N}^{n}\right\}$, то существуют, во-первых, обобщенная функиия $\Phi(e) \in \mathscr{S}^{\prime}\left(S^{n-1}\right)$, для которой при любом $j$, удовлетворяющем условию $(j, a)=-\alpha-|a|$, выполнено равенство

$$
\left(\Phi(e), E_{j}(e)\right)=0
$$

$u$ во-вторых, постоянные $A_{m}$, где мультииндекс $m \in \mathbb{Z}_{+}^{n},(m, a)=-\alpha-|a|$, такие, что

$$
\begin{array}{r}
(f(t), \varphi(t))=\int_{0}^{\infty} r^{\alpha+|a|-1}\left(\Phi(e),\left(\varphi\left(r^{a_{1}} e_{1}, \ldots, r^{a_{n}} e_{n}\right)\right.\right. \\
\left.\left.-\sum_{\substack{(m, a)<-\alpha-|a| \\
m \in \mathbb{Z}_{+}^{n}}} r^{(m, a)} \frac{1}{m !} \varphi^{(m)}(0) E_{m}(e)\right)\right)_{e} d r \\
+\sum_{\substack{(m, a)=-\alpha-|a| \\
m \in \mathbb{Z}_{+}^{n}}} A_{m} \varphi^{(m)}(0) \quad \forall \varphi(t) \in \mathscr{S}\left(\mathbb{R}^{n}\right) .
\end{array}
$$

Нетрудно показать, что обобщенная функция $f(t) \in \mathscr{S}^{\prime}\left(\mathbb{R}^{n}\right)$ квазиоднородна степени $\alpha$ относительно группы преобразований аргумента, определяемой вектором $a=\left(a_{1}, \ldots, a_{n}\right) \in \mathbb{R}_{+}^{n}$, только тогда, когда она удовлетворяет уравнению

$$
\sum_{i=1}^{n} a_{i} t_{i} \frac{f(t)}{d t_{i}}=\alpha f(t)
$$

так что утверждение 4.3 дает представление обобщенных функций, удовлетворяющих обобщенному уравнению Эйлера (4.19).

\section{§ 5. Некоторые применения полученных результатов}

В этом параграфе мы рассмотрим обобщенные функции из $\mathscr{S}^{\prime}\left(\mathbb{R}^{n}\right)$, асимптотически однородные в нуле по группе преобразований, определяемой вектором $a$ (см. определение 0.2 ), и изучим их связь с особенностями функций, голоморфных в трубчатых областях над ортантами.

Пусть $f(z) \in H\left(T^{C}\right)$, где $C$ - острый, выпуклый, открытый конус в $\mathbb{R}^{n}$, а $T^{C}=\mathbb{R}^{n}+i C$ - трубчатая область над конусом $C$. Это означает, что $f(z)$ голоморфна в $T^{C}$ и удовлетворяет в этой области оценке

$$
|f(z)| \leqslant A \frac{(1+|z|)^{d}}{\Delta_{C}^{b}(y)}, \quad z=x+i y, \quad x \in \mathbb{R}^{n}, \quad y \in C,
$$

с некоторыми постоянными $A, b, d$. Здесь $\Delta_{C}(y)-$ расстояние от $y \in C$ до границы конуса $C$ (см. [7]).

Известно, что у $f(z)$ существует граничное значение в $\mathscr{S}^{\prime}$ при $y \rightarrow+0$, так что

$$
f(x)=b \cdot v \cdot f(z)=\lim _{y \rightarrow+0} f(x+i y), \quad y \in C^{\prime},
$$

где $C^{\prime} \Subset C$. 
В качестве конусов $C$ далее будут служить ортанты $\left\{ \pm y_{i}>0\right\}$ в $\mathbb{R}^{n}$. Их $2^{n}$ штук. Перенумеруем их и обозначим $\ell$-й ортант через $\mathbb{R}_{\ell}, \ell=1,2, \ldots, 2^{n}$.

Отметим, что расстояние от $y \in \mathbb{R}_{\ell}^{n}$ до границы ортанта $\triangle_{\mathbb{R}_{\ell}^{n}}(y)=\min \left\{\left|y_{i}\right|\right.$, $i=1, \ldots, n\}$. Кроме того, для любого вектора $a=\left(a_{1}, \ldots, a_{n}\right) \in \mathbb{R}_{+}^{n}$ группа $\left\{U_{k}^{-a}, k>0\right\}$ оставляет инвариантной каждую из трубчатых областей $T^{\mathbb{R}_{\ell}^{n}}$. Это позволяет ввести следующее

ОПРЕДЕЛЕНИЕ 5.1. Пусть $\rho(k)$ - автомодельная функция и $a=\left(a_{1}, \ldots, a_{n}\right)-$ вектор из $\mathbb{R}_{+}^{n}$. Положим $z=|z| \zeta$, где $\zeta=\left(\zeta_{1}, \ldots, \zeta_{n}\right),|\zeta|=1$. Будем говорить, что голоморфная функция $f(z) \in H\left(T^{\mathbb{R}_{\ell}^{n}}\right)$ ведет себя в нуле вдоль траекторий группы $\left\{U_{k}^{-a}\right\}$ как $\rho(k)$, если

1) существует функция $g_{\ell}(\zeta)$ такая, что

$$
\lim _{k \rightarrow+\infty} \frac{1}{\rho(k)} f\left(U_{k}^{-a} \zeta\right)=g_{\ell}(\zeta) \quad \forall \zeta \in T^{\mathbb{R}_{\ell}^{n}} \cap\{|\zeta|=1\} ;
$$

2) существуют числа $b$ и $c$ такие, что

$$
\left|\frac{1}{\rho(k)} f\left(U_{k}^{-a} \zeta\right)\right| \leqslant \frac{c}{\varphi^{b}}, \quad \zeta \in T^{\mathbb{R}_{\ell}^{n}} \cap\{|\zeta|=1\},
$$

где $\varphi=\min _{i}\left\{\left|\operatorname{Im} \zeta_{i}\right|, i=1,2, \ldots, n\right\}$, т.е. $\varphi$ - это расстояние от точки на единичной сфере, определяемой вектором $\operatorname{Im} \zeta$, до границы ортанта $\mathbb{R}_{\ell}^{n}$.

При этом будем писать

$$
f\left(U_{k}^{-a} z\right) \sim \rho(k) g_{\ell}(\zeta), \quad k \sim \infty, \quad \zeta \in T^{\mathbb{R}_{\ell}^{n}} \cap\{|\zeta|=1\} .
$$

Имеет место следующее

УтвеРЖДЕНИЕ 5.1. Пусть $f(z) \in H\left(T^{\mathbb{R}_{q}^{n}}\right)$ u $f(x)=$ b.v.f(z). Для того чтобъ $f(x) \in A O_{\rho}^{-a}\left(\mathscr{S}\left(\mathbb{R}^{n}\right)\right)$, необходимо и достаточно, чтобъ

$$
f\left(U_{k}^{-a} z\right) \sim \rho(k) g_{q}(\zeta), \quad k \sim \infty, \quad \zeta \in T^{\mathbb{R}_{q}^{n}} \cap\{|\zeta|=1\} .
$$

Доказательство непосредственно следует из общей тауберовой теоремы (см. $[4 ; \S 4.2])$. Для этого надо применить к $f(x)$ преобразование Фурье и учесть, что

$$
\frac{1}{\rho_{1}(k)} F\left[f\left(U_{k}^{a} t\right)\right](x)=\frac{1}{\rho(k)} F[f(t)]\left(U_{k}^{-a} x\right) .
$$

Здесь $F[\cdot]$ означает преобразование Фурье и $\rho(k)=k^{|a|} \rho_{1}(k)$.

TeOpema 5.1. Пусть $f(x) \in \mathscr{S}^{\prime}\left(\mathbb{R}^{n}\right), n>1, u f(x) \in A O_{\rho}^{-a}\left(\mathscr{S}\left(\mathbb{R}^{n}\right)\right)$, где вектор а $\in \mathbb{R}_{+}^{n}$. Тогда существуют

$$
f_{\ell}(z) \in H\left(T^{\mathbb{R}_{\ell}^{n}}\right), \quad \ell=1,2, \ldots, 2^{n},
$$

такие, что

$$
f_{\ell}\left(U_{k}^{-a} z\right) \sim \rho(k) g_{\ell}(\zeta), \quad k \sim \infty, \quad \zeta \in T^{\mathbb{R}_{\ell}^{n}} \cap\{|\zeta|=1\},
$$

причем

$$
f(x)=\sum_{\ell} b \cdot v \cdot f_{\ell}(z)
$$


ЗАмЕчАниЕ. При $n=1$, как будет доказано далее, утверждение теоремы 5.1 останется справедливым для некритических порядков автомодельности $\rho(k)$, т.е. для $\alpha \neq 0,-1,-2, \ldots$. Отметим, что в этом случае без ограничения общности можно считать что $a=1$.

ДокАЗАТЕЛЬство. Перейдем к обратному преобразованию Фурье: $\widetilde{f}(t)=$ $F[f(x)](t)$. Отметим, что $\widetilde{f}(t) \in A O_{\rho_{1}}^{a}\left(\mathscr{S}\left(\mathbb{R}^{n}\right)\right)$, где $\rho_{1}(k)=k^{-|a|} \rho(k)$. Достаточно доказать, что $\widetilde{f}(t)$ можно представить в виде суммы $\sum_{\ell} \widetilde{f}_{\ell}(t)$, где $\widetilde{f}_{\ell}(t) \in A O_{\rho_{1}}^{a}\left(\mathscr{S}\left(\mathbb{R}^{n}\right)\right)$ и $\operatorname{supp} \widetilde{f}_{\ell}(t) \subset \overline{\mathbb{R}}_{\ell}^{n}$ (см. утверждение 5.1).

Считая, что порядок $\rho(k)$ равен $\alpha$, рассмотрим два случая.

1) $\alpha \neq-\left(a_{1} n_{1}+\cdots+a_{n} n_{n}\right)$, где $n_{i}=0,1,2, \ldots ; i=1,2, \ldots, n$. Тогда согласно теореме 4.1 (см. (4.2)-(4.5)) $\widetilde{f}(t)=f_{1}(t)+f_{0}(t)$, где $f_{0}(t)$ имеет компактный носитель и достаточно много моментов, равных нулю. Пусть, например,

$$
\left(f_{0}(t), t^{j}\right)=0, \quad|j| \leqslant N
$$

с некоторым $N$. Функция $f_{1}(t)$ представлена формулой $(4.5)$, с некоторым $\nu$ таким, что $-\nu-\alpha \notin \mathbb{Z}_{+}$, где функция $\gamma(r, e)$ непрерывна по $r$ со значениями в $\mathscr{S}^{\prime}\left(S^{n-1}\right)$ и

$$
\gamma(r, e) \sim r^{\nu+N-1} \rho(r) \Psi(e), \quad r \rightarrow \infty,
$$

для некоторой $\Psi(e) \in \mathscr{S}^{\prime}\left(S^{n-1}\right)$. Так как функция $\gamma(r, e)$ непрерывна по $r$, то она разбивается на сумму $\sum_{\ell=1}^{2^{n}} \gamma^{\ell}(r, e)$ измеримых по $r$ функций со значениями в $\mathscr{S}^{\prime}\left(S^{n-1}\right)$, имеющих ту же асимптотику при $r \rightarrow+\infty$ и $\operatorname{supp} \gamma^{\ell}(r, e) \subset$ $S^{n-1} \cap \mathbb{R}_{\ell}^{n}$ при любом $r>1$. (См. доказательство теоремы 6.1 и теоремы П2 работы [8], в которых использованы результаты работ [9], [10].) Обозначим через $f_{1 \ell}(t)$ обобщенную функцию, определяемую формулой $(4.5)$, в которую вместо $\gamma(r, e)$ подставлена функция $\gamma^{\ell}(r, e)$ с индексом $\ell$.

Далее, обобщенную функцию $f_{0}(t)$ представим в виде

$$
f_{0}(t)=\sum_{\ell} f_{0 \ell}(t), \quad \text { где } \operatorname{supp} f_{0 \ell}(t) \subset \mathbb{R}_{\ell}^{n} \cap\{|t| \leqslant 1\} .
$$

Подправим $f_{0 \ell}$ линейными комбинациями дельта-функций и их производных так, чтобы для каждой из них выполнялось условие (5.7). Для этого подберем числа

$$
c_{m}^{1}, \quad m=\left(m_{1}, \ldots, m_{n}\right), \quad|m| \leqslant M
$$

(с достаточно большим $M$ ), так, чтобы

$$
\left(f_{01}(t)+\sum_{|m|=1}^{M} c_{m}^{1} \delta^{(m)}(t), t^{j}\right)=0, \quad|j| \leqslant N .
$$

Положим

$$
\widetilde{f}_{01}(t)=f_{01}(t)+\sum_{|m|=1}^{M} c_{m}^{1} \delta^{(m)}(t)
$$


Предположим, что для $k=1,2, \ldots, \ell-1,1<\ell<2^{n}$, числа $c_{m}^{k}$ уже выбраны и функции $\widetilde{f}_{0 k}(t)$ уже построены. Подберем числа $c_{m}^{\ell}$ так, чтобы

$$
\left(f_{0 \ell}(t)-\sum_{|m|=1}^{M} c_{m}^{\ell-1} \delta^{(m)}(t)+\sum_{|m|=1}^{M} c_{m}^{\ell} \delta^{(m)}(t), t^{j}\right)=0 .
$$

Положим

$$
\begin{gathered}
\widetilde{f}_{0 \ell}(t)=f_{0 \ell}(t)-\sum_{|m|=1}^{M} c_{m}^{\ell-1} \delta^{(m)}(t)+\sum_{|m|=1}^{M} c_{m}^{\ell} \delta^{(m)}(t), \quad 1<\ell<2^{n}, \\
\tilde{f}_{02^{n}}(t)=f_{02^{n}}-\sum_{|m|=1}^{M} c_{m}^{2^{n}-1} \delta^{(m)}(t) .
\end{gathered}
$$

Очевидно, что для $\widetilde{f}_{0 \ell}$ выполнены соотношения (5.7) и (5.9), а именно,

$$
\left(\widetilde{f}_{0 \ell}(t), t^{j}\right)=0, \quad|j| \leqslant N, \quad f_{0}(t)=\sum_{\ell} \widetilde{f}_{0 \ell}(t) .
$$

Далее, положим $\widetilde{f}_{\ell}(t)=f_{1 \ell}(t)+\widetilde{f}_{0 \ell}(t)$. По теореме 4.1 (см. также замечание к теореме 4.2) $\widetilde{f}_{\ell} \in A O_{\rho_{1}}^{a}\left(\mathscr{S}\left(\mathbb{R}^{n}\right)\right)$ и ее носитель принадлежит $\overline{\mathbb{R}}_{\ell}^{n}$. Теорема в случае 1$)$ доказана, в том числе и при $n=1$.

2) Пусть теперь $\alpha \in\left\{-\left(a_{1} n_{1}+\cdots+a_{n} n_{n}\right), n_{i}=0,1,2, \ldots, i=1,2, \ldots, n\right\}$. В этом случае согласно теореме 4.2 (см. (4.9)-(4.15)) имеем $\tilde{f}(t)=f_{0}(t)+$ $f_{1}(t)+f_{2}(t)$, где $f_{0}(t)$ имеет компактный носитель и достаточно много моментов, равных нулю (см. (5.7)). Функция $f_{1}(t)$ представлена формулой $(4.12)$, где функция $\gamma(r, e)$ непрерывна по $r$ со значениями в $\mathscr{S}^{\prime}\left(S^{n-1}\right)$, с некоторым $\nu$ таким, что $-\nu-\alpha \in \mathbb{Z}_{+}$, и имеет асимптотику, определяемую формулой (5.8). Функция $f_{2}(t)$ представлена формулой (4.15), где функция $\omega(r, e)$ непрерывна по $r$ со значениями в $\mathscr{S}^{\prime}\left(S^{n-1}\right)$, с некоторым $\mu$ таким, что $-\mu-\alpha \notin \mathbb{Z}_{+}$и имеет асимптотику, определяемую формулой

$$
\omega(r, e) \sim r^{\mu+N-1} \rho(r) \Phi(e), \quad r \rightarrow \infty,
$$

с некоторой $\Phi(e) \in \mathscr{S}^{\prime}\left(S^{n-1}\right)$, и ортогональна пространству $\mathscr{E}_{-\alpha}^{a}$, т.е.

$$
(\omega(r, e), \varphi(e)) \equiv 0 \quad \forall \varphi(e) \in \mathscr{E}_{-\alpha}^{a} .
$$

$\mathrm{C}$ функциями $f_{0}$ и $f_{1}$ поступаем точно так же, как и в предыдущем случае, т.е. находим $f_{0 \ell}(t)$ и $f_{1 \ell}(t)$ с носителем в $\overline{\mathbb{R}}_{\ell}^{n}$ соответственно, причем каждая $f_{0 \ell}$ имеет компактный носитель и многие ее моменты равны нулю (см. (5.7)). С другой стороны, по той же теореме $4.2 f_{1 \ell}(t) \in A O_{\rho_{1}}^{a}\left(\mathscr{S}\left(\mathbb{R}^{n}\right)\right)$ и выполнены соотношения

$$
f_{0}(t)=\sum_{\ell} f_{0 \ell}(t), \quad f_{1}(t)=\sum_{\ell} f_{1 \ell}(t)
$$

Для функции $f_{2}(t)$ покажем, что она представляется в виде суммы $\sum_{\ell} \widetilde{f}_{2, \ell}(t)$, где

$$
\operatorname{supp} \widetilde{f}_{2, \ell} \subset \overline{\mathbb{R}}_{\ell}^{n}, \quad \widetilde{f}_{2, \ell}(t) \in A O_{\rho_{1}}^{a}\left(\mathscr{S}\left(\mathbb{R}_{\ell}^{n}\right)\right) .
$$


Действительно, так как $\omega(r, e)$ непрерывна по $r$, то она разбивается на сумму $\sum_{\ell=1}^{2^{n}} \omega^{\ell}(r, e)$ измеримых по $r$ функций со значениями в $\mathscr{S}^{\prime}\left(S^{n-1}\right)$, имеющих ту же асимптотику при $r \rightarrow+\infty$ и $\operatorname{supp} \omega^{\ell}(r, e) \subset S^{n-1} \cap \mathbb{R}_{\ell}^{n}$ при любом $r$. Подправим функции $\omega^{\ell}$ так, чтобы для каждой из них выполнялись соотношения (5.13).

Для этого предварительно заметим, что пространство $\mathscr{E}_{-\alpha}^{a}$ конечномерно. Пусть $\left\{E_{s}(e), s=1, \ldots, M\right\}-$ его базис. Покажем, что для любой точки $e_{0} \in S^{n-1}$ существуют обобщенные функции $A_{s}(e) \in \mathscr{S}^{\prime}\left(S^{n-1}\right)$, сосредоточенные в точке $e_{0}$ на сфере (т.е. линейные комбинации $\delta\left(e-e_{0}\right)$ и их производных в некоторой заранее выбранной карте в окрестности точки $\left.e_{0}\right)$, такие, что

$$
\left(A_{\tau}(e), E_{s}(e)\right)=\delta_{\tau s}, \quad \tau, s=1,2, \ldots, M
$$

Можно также считать, что в этой карте все $E_{s}(e)$ аналитичны.

Действительно, зафиксируем точку $e_{0}$ на сфере и найдем число $N$ такое, чтобы $M$ векторов $\left\{E_{s}^{(i)}\left(e_{0}\right),|i| \leqslant N\right\}, s=1, \ldots, M$, были линейно независимы в $\mathbb{C}^{n}$. Такое $N$ найдется, так как в противном случае для любого $N$ нашелся бы вектор $C^{N}=\left(c_{1}^{N}, \ldots, c_{M}^{N}\right),|C|=1$, такой, что

$$
\sum_{s=1}^{M} c_{s}^{N}\left\{E_{s}^{(i)}\left(e_{0}\right)\right\}=0 \quad \forall|i| \leqslant N .
$$

Выбирая из последовательности $\left\{C^{N}, N=1,2, \ldots,\right\}$ некоторую сходящуюся подпоследовательность (например сходящуюся к $C=\left(c_{1}, \ldots, c_{n}\right)$ ), получим, что все производные ненулевой функции $\psi(e)=\sum_{s=1}^{M} c_{s} E_{s}(e)$, аналитической в окрестности $e_{0}$ на сфере, обращаются в нуль, что невозможно.

Теперь поскольку в матрице $\left\{E_{s}^{(i)}\left(e_{0}\right)\right\}, s=1, \ldots, M,|i| \leqslant N$, в силу линейной независимости строк существует минор $M \times M$ с детерминантом, отличным от нуля, то линейной заменой он приводится к единичной матрице. Таким образом, найдутся числа $c_{r, i}$ такие, что

$$
\sum_{|i| \leqslant N} c_{r, i} E_{s}^{(i)}\left(e_{0}\right)=\delta_{r s}, \quad r, s=1,2, \ldots, M
$$

что и доказывает (5.14).

Поскольку $n>1$, то без ограничения общности можно считать, что ортанты с соседними номерами имеют хотя бы один общий луч. Для каждого $\ell=$ $1,2, \ldots, 2^{n}-1$ фиксируем луч, принадлежащий $\overline{\mathbb{R}}_{\ell}^{n} \cap \overline{\mathbb{R}}_{\ell+1}^{n}$, и точку $e_{0}^{\ell} \in S^{n-1}-$ точку пересечения этого луча с единичной сферой. Пусть $A_{\tau}^{\ell}(e) \in \mathscr{S}^{\prime}\left(S^{n-1}\right)$, $\tau=1, \ldots, M,-$ обобщенные функции, при каждом $\ell$ удовлетворяющие соотношениям (5.17) с точкой $e_{0}$ равной $e_{0}^{\ell}$. Далее положим

$$
\left\{\begin{array}{l}
\widehat{\omega}^{1}(r, e)=\omega^{1}(r, e)-\mathscr{D}_{1}(r, e), \\
\widehat{\omega}^{\ell}(r, e)=\omega^{\ell}(r, e)+\mathscr{D}_{\ell-1}(r, e)-\mathscr{D}_{\ell}(r, e), \quad 2 \leqslant \ell \leqslant 2^{n}-1, \\
\widehat{\omega}^{2^{n}}(r, e)=\omega^{2^{n}}(r, e)+\mathscr{D}_{2^{n}-1}(r, e),
\end{array}\right.
$$


где

$$
\begin{gathered}
\mathscr{D}_{1}(r, e)=\sum_{\tau=1}^{M}\left(\omega^{1}(r, e), E_{\tau}(e)\right) A_{\tau}^{1}(e), \\
\mathscr{D}_{\ell}(r, e)=\sum_{\tau=1}^{M}\left(\omega^{\ell}(r, e)+\mathscr{D}_{\ell-1}(r, e), E_{\tau}(e)\right) A_{\tau}^{\ell}(e), \quad \ell=2, \ldots, 2^{n}-1 .
\end{gathered}
$$

Нетрудно видеть, что по построению

$$
\sum_{\ell=1}^{2^{n}} \widehat{\omega}^{\ell}(r, e)=\omega(r, e), \quad \operatorname{supp} \widehat{\omega}^{\ell}(r, e) \subset \overline{\mathbb{R}}_{\ell}^{n}, \quad \ell=1,2, \ldots, 2^{n},
$$

и, кроме того, выполнено условие ортогональности

$$
\left(\widehat{\omega}^{\ell}(r, e), E_{s}(e)\right) \equiv 0, \quad s=1, \ldots, M,
$$

для $\ell=1,2, \ldots, 2^{n}-1$ в силу (5.14) и для $\ell=2^{n}$ в силу (5.13). Очевидно также, что для $\widehat{\omega}^{\ell}(r, e)$ выполнено асимптотическое соотношение, аналогичное (5.12). Теперь соотношение (4.15), куда вместо $\omega(r, e)$ подставлено $\widehat{\omega}^{\ell}(r, e)$, согласно теореме 4.2 (см. также замечание к ней) дает обобщенную функцию $f_{2 \ell}(t) \in A O_{\rho_{1}}^{a}\left(\mathscr{S}\left(\mathbb{R}^{n}\right)\right)$ с носителем в $\overline{\mathbb{R}}_{\ell}^{n}$. Полагая $\widetilde{f}_{\ell}(t)=f_{0 \ell}(t)+f_{1 \ell}(t)+f_{2 \ell}(t)$, завершаем доказательство теоремы.

\section{Список литературы}

[1] Е. Сенета, Правильно меняющиеся функиии, Наука, М., 1985; пер. с англ.: Eu. Seneta, Regularly varying functions, Lecture Notes in Math., 508, SpringerVerlag, Berlin-Heidelberg-New York, 1976.

[2] O. von Grudzinski, Quasihomogeneous distributions, North-Holland Math. Stud., 165, North-Holland, Amsterdam, 1991.

[3] Ю.Н. Дрожжинов, Б. И. Завьялов, "Асимптотически однородные обобщенные функции в сферическом представлении и некоторые применения", Докл. РАН, 405:1 (2005), 18-21; англ. пер.: Yu. N. Drozhzhinov, B. I. Zav'yalov, "Asymptotically homogeneous generalized functions in spherical representation and applications", Dokl. Math., 72:3 (2005), 839-542.

[4] В. С. Владимиров, Ю.Н. Дрожжинов, Б. И. Завьялов, Многомерные тауберовы теоремы для обобщенных функиий, Наука, М., 1986.

[5] Х. Шефер, Топологические векторные пространства, Мир, М., 1971; пер. с англ.: H. H. Schaefer, Topological vector spaces, Macmillan, New York-London, 1966.

[6] И. М. Гельфанд, Г. Е. Шилов, Обобщенные функиии и действия над ними, вып. 1, Физматгиз, М., 1958; англ. пер.: I. M. Gel'fand, G. E. Shilov, Generalized functions. Vol. I: Properties and operations, Academic Press, New York-London, 1964.

[7] В. С. Владимиров, Обобщенные функиии в математической физике, Наука, М., 1979; англ. пер.: V.S. Vladimirov, Generalized functions in mathematical physics, Mir, Moscow, 1979.

[8] Ю.Н. Дрожжинов, Б. И. Завьялов, "Асимптотически однородные обобщенные функции и граничные свойства функций, голоморфных в трубчатых конусах", Изв. РАН. Сер. матем., 70:6 (2006), 45-92; англ. пер.: Yu. N. Drozhzhinov, B. I. Zav'yalov, "Asymptotically homogeneous generalized functions and boundary properties of functions holomorphic in tubular cones", Izv. Math., 70:6 (2006), $1117-1164$. 
[9] А.Н. Колмогоров, С. В. Фомин, Элементы теории функиий и функиионального анализа, 4-е изд., Наука, М., 1976; англ. пер. 2-го изд.: А. N. Kolmogorov, S. V. Fomin, Introductory real analysis, Prentice-Hall, Englewood Cliffs, NJ, 1970.

[10] В. С. Владимиров, Методы теории функиии многих комплексных переменных, Наука, M., 1964; англ. пер.: V.S. Vladimirov, Methods of the theory of functions of many complex variables, Cambridge, MA-London, MIT Press, 1966.

Ю. Н. Дрожжинов (Yu. N. Drozhzhinov)

Математический институт им. В. А. Стеклова РАН

E-mail: drozzin@mi.ras.ru

Б. И. Завьялов (В. I. Zav'yalov)

Математический институт им. В. А. Стеклова РАН

E-mail: bzavial@mi.ras.ru
Поступила в редакцию 17.04.2008 и 27.11.2008 